\title{
Estimating the burden of illness related to genital warts in the Philippines: a nationally representative cross-sectional study
}

Lani Buenconsejo ${ }^{1}$, Smita Kothari-Talwar ${ }^{2 *}$, Karen Yee ${ }^{3}$, Amit Kulkarni ${ }^{2}$, Nuria Lara ${ }^{4}$, Montserrat Roset ${ }^{4}$, Anna R. Giuliano ${ }^{5}$ and Suzanne Garland ${ }^{6}$

\begin{abstract}
Background: This study estimated genital warts prevalence, genital-warts-related healthcare resource use and costs, and self-reported human-papillomavirus-related psychosocial impact among male and female patients aged 18-60 years in the Philippines.

Methods: Prevalence was estimated using daily logs numbering genital warts patients treated by participating physicians in 4 Philippine regions over a 5-week period (09JUL2011-24SEP2012). Physicians also completed a survey assessing patient referral patterns, healthcare resource use, treatment, and follow-up care. Psychosocial impact was estimated using the human papillomavirus impact profile and the EQ-5D questionnaires. HIP and EQ-5D scores were compared according to the presence of GW (males) and HPV disease (females). CECA scores were also compared by gender and age groups.
\end{abstract}

Results: Overall genital warts prevalence was estimated at 4.78\% (95\% confidence interval [Cl]: 4.58-4.98\%) for men and women aged 18-60 years. Genital warts prevalence was 3.39\% (95\% Cl: 3.13-3.65\%) and 8.0\% (95\% Cl: 7.69-8.31\%) among women and men, respectively. Prevalence estimates were highest in infectious disease specialist practices $18.67 \%$ (95\% Cl: 18.66-18.69\%). Two thirds of the 233 (69.14\%) male and 166 (67.20\%) female patients were newlydiagnosed genital warts cases. Median costs for genital warts diagnosis and treatment reached 7121 and 7000 Philippine pesos among men and women, respectively. In the Cuestionario Específico para Condiloma Acuminado questionnaire, no statistically significant differences between patients were observed. In the EQ-5D questionnaire, male genital warts patients reported lower mean visual analogue scale scores than those without genital warts (78.20 vs $86.34, p<0.0001$ ). Mean visual analogue scale score values and utility values were lower for women with human-papillomavirus-related diseases than those without (77.98 vs 78.93, and 0.84 vs 0.88 , respectively).

Conclusions: Genital warts is prevalent in the Philippines; more than $60 \%$ of cases were newly diagnosed, contributing to high genital-warts-related healthcare resource costs. Diagnosis of genital warts and human papillomavirus negatively impacted psychosocial indices such as patient well-being and health-related quality of life.

Keywords: Genital warts, Prevalence, Health care cost and utilization, Psychosocial impact

\footnotetext{
* Correspondence: publications@statinmed.com

KY was an employee of Merck \& Co. Inc. at the time of the study.

${ }^{2}$ Merck \& Co. Inc., Kenilworth, NJ, USA

Full list of author information is available at the end of the article
}

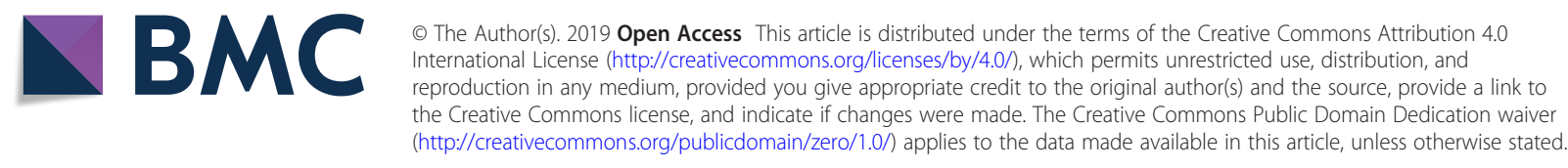




\section{Background}

Human papillomavirus (HPV) causes one of the most common sexually transmitted infections (STIs) [1]. HPV occurs soon after sexual debut and is mostly prevalent in young adults [2]. The more than 130 identified types of this virus are divided into 2 groups according to their epidemiological association with cervical cancer [3]. Low-grade HPV includes subtypes 6 and 11, which are estimated to cause approximately $90 \%$ of genital warts (GW) cases [1, 4]. High-risk HPV, including HPV subtypes 16 and 18, induces precancerous lesions such as cervical intraepithelial neoplasia (CIN), cervical cancer, and anogenital cancer [5].

Research suggests that an estimated 6.2 million new GW infections occur annually in individuals aged 14-44 years [6]. However, national incidence data by country is limited, and prevalence estimates by country range widely, from $0.7 \%$ in South Korea to up to $25.6 \%$ in Nigeria [7-9]. The US National Health and Nutrition Examination Survey found that from 1999 to 2004, 5.6\% of survey respondents, aged 18-59 years, self-reported a GW diagnosis [10]. The percentage was higher in women than men: (7.2\%; 95\% confidence interval [CI]: $6.2-8.4 \%)$ vs. (4\%; 95\% CI: 3.2-5.0\%), respectively [10]. A 2015 study using data compiled from regional and national registries from 32 European countries found the estimated annual number of new GW cases ranged between 755,937 and 938, 212 in 2015 [11]. In Australia, GW incidence was estimated at 2.19 cases per 1000 with a lifetime prevalence of approximately $4 \%$ among Australians aged 16 to 59 years [12-14]. GW incidence has been estimated at 126 per 100,000 person-years in Canada, 203.7 per 100,000 persons-years in Hong Kong, 113.7 per 100,000 personyears in Germany, and 118 per 100,000 person-years in Spain [15]. In Spain, the overall estimated prevalence of GW was 182/100,000 (population) [7].

GW treatment and management weigh heavily on healthcare systems due to the large economic burden associated with repeated physician visits, medication application, and mechanical removal of the warts through cryotherapy [6]. GW treatments can result in significant direct and indirect costs, and with an expected increase in GW incidence, the economic burden is also likely to increase. Two studies evaluating the economic burden of GW in Spain and Germany found similar conclusions related to overall costs. In Germany, overall third party payer (TPP) costs were $€ 49$ million and total societal costs were $€ 54.1$ million [16]; in Spain, TPP costs were estimated at $€ 47$ million and total societal costs at $\sim 660$ million [7]. In Australia, the annual healthcare costs associated with GW management were over A \$14 million, with an estimated cost per treated case of $\sim$ A $\$ 250$ and $\$ 385$ for men and women, respectively [12]. Furthermore, several studies have shown that GW had a negative psychosocial impact on well-being and health related quality of life (HRQL) [17-19]. Patients with GW experienced heavier psychosocial burden than the general population, and females experienced higher burden than males. HRQL dimensions highly impacted for GW depended on the questionnaire used, but those related with self-image and sexual impact were usually highly impacted [19, 20].

To date in the Philippines, limited data exist on the actual epidemiology of GW within the general population due to a lack of research on GW incidence and prevalence. The current data available in the Philippines primarily focuses on cervical cancer where HPV has shown a strong association. In a 1995-98 case study among Filipino cervical cancer cases, the prevalence of all HPV types in the cases was 93.5\% [21]. Another study in the Philippines examining the prevalence of HPV infection and cervical abnormalities among 369 female commercial sex workers estimated HPV infection at $57.2 \%$ [22]. As such, it is likely that the burden of HPV-related GW is comparatively high in the Philippines, thereby causing a greater economic impact on society and psychosocial burden on individuals.

The aim of this study was to estimate GW prevalence, GW-related healthcare resource use and costs, as well as the self-reported disease-related psychosocial impact among male and female patients aged 18-60 years in the Philippines.

\section{Methods \\ Study design}

This was a cross-sectional study conducted to estimate GW prevalence in a 2 -week period using physician surveys to approximate healthcare resource use, a daily physician log to assess prevalence, and patient surveys to estimate the psychosocial impact of GW among men and women (18-60 years) in four major cities in the Philippines (Manila, Luzon, Mindanao, and Visayas).

\section{Prevalence, healthcare costs, and resource use}

Referral patterns, healthcare resource use, and costs for GW patients were captured through a 30-min face-toface physician survey between 09JUL2011-24SEP2012. The survey included questions related to healthcare resource use as part of the usual course of diagnoses, inoffice and at-home treatment and procedures, and follow-up care (medical visits, emergency room [ER] visits, hospitalizations). In addition, several survey questions were included to also determine patient referral patterns within the practice, from general practitioners (GPs) to specialists and between specialists.

Secondly, participant physicians completed a daily log during a 2-week period, recording information on the number of patients seen for all causes, including their age and gender; the number of GW patients seen for new or existing disease episodes (whether GW was the primary reason for the visit or was incidentally diagnosed at the time of 
visit); and the number of patients retained for treatment versus those referred to another specialist for treatment or for follow-up with the general practitioner.

Participating physicians were identified through the Intercontinental Marketing Services (IMS) database in the Philippines, which includes information on approximately 11,344 physicians. For this study, physician locations were selected to reflect all major regions of the country, including the metropolitan area of Manila and key cities in the Luzon, Visayas, and Mindanao regions. The physicians were evenly divided between the public and private sectors.

Physicians were included if they:

- were primary care physicians (PCPs), GPs or family medicine (FM) physicians, obstetrician/gynecologists (OB/GYNs), urologists (UROs), dermatologists (DERMs), or infectious disease specialists (IDSs) with 2-30 years of practice experience;

- devoted $\geq 50 \%$ of their time to treating patients for outpatient visits, $\geq 3$ work days per week;

- (GPs or OB/GYNs) typically handled $\geq 50$ outpatient visits per week;

- (UROs) typically handled $\geq 30$ outpatient visits per week;

- (IDSs or DERMs) typically handled $\geq 20$ outpatient visits per week; and

- treated patients aged $18-60$ years for $\geq 50 \%$ of their outpatient visits.

\section{Psychosocial impact of GW and selected HPV diseases}

Physicians who completed the physician survey and the patient daily $\log$ were invited to participate in the second phase of the study if they were treating $a \geq 3$ patients with GW per month (all specialists) and $\geq 1$ patient per month with $\mathrm{CIN} 2 / 3$ (only $\mathrm{OB} / \mathrm{GYNs}$ ). Patients attending to the study investigators office were consecutively invited to participate in a one-time survey and giving them a patient informed consent form with a short description of the study.

Female patients participating in the psychosocial impact analysis were selected based on their new or existing HPV-related outcomes or diagnoses (excluding patients with cervical cancer) within 3 months prior to study recruitment. Women were included if they:

- were aged 18-60 years;

- experienced an HPV-related event within the past 3 months;

- were in good self-reported health; and

- had one of the following:

abnormal Papanicolaou (Pap) test results with no definitive histology, conforming to the Bethesda Category-2001 category of squamous or glandular cell abnormality, and no previous high-risk HPV test performed;

positive high-risk HPV DNA test results after an abnormal Pap test, as defined above;

a diagnosis of external GW or treatment for recurrence;

a histological diagnosis of a precancerous or cancerous cervical lesion (eg, CIN1, CIN2, CIN3);

(control group) a normal Pap result with no abnormal Pap test or definitive therapy within the past year; or

$\geq 2$ of the above conditions (not including GW patients) categorized as upper level disease severity.

Male patients participating in the psychosocial impact analysis had a new or existing external GW case (ie, GW, anal warts, venereal warts) within 3 months prior to study recruitment. Male patients included in the study were aged 18-60 years, were in good self-reported physical health, and belonged to one of the following categories:

a) had new or existing external GW within the past 3 months of study recruitment; or

b) (control group) did not have a GW diagnosis, GW treatment prescription, or surgery or therapy in the genital area.

Patients were excluded if they were females diagnosed with GW with precancerous cervical lesions, abnormal Pap test results, and HPV-positive test results; had evidence of the presence of any other concurrent/active STI; were concurrently enrolled in clinical studies of investigational agents; had a history of known prior vaccination with HPV vaccine; had recent ( $\leq 1$ year from enrollment date) or ongoing alcohol or other substance abuse; or were unable to give informed consent.

All data collection was conducted at the workplaces of the participating physicians. To measure psychosocial burden (general health, sexual activity, cervical cancer screening behavior, psychosocial impact, $\mathrm{GW}$ experience, sociodemographic information), participants completed the HPV impact profile (HIP), the Cuestionario Específico para Condiloma Acuminado (CECA-specific questionnaire for condylomata acuminata), and the EuroQol-5 Dimension 3 level (EQ-5D) questionnaire, which were translated to Filipino (standardized Tagalog) and culturally pre-tested.

The HIP is a validated 29-item self-administered questionnaire, designed to measure the psychosocial impact of HPV-related health conditions in women [23]. HIP mean scores are categorized as $<40$ (no/little impact), 40-70 (moderate impact), and $>70$ (high psychological impact). This survey was adapted for use in males in consultation with the original developer and underwent cognitive testing in the United States. 
The CECA questionnaire includes 10 questions across 2 domains: emotional and sexual activity [24, 25]. CECA scores range from 0 (worst HRQL) to 100 (best HRQL).

The EQ-5D is a 2-part questionnaire, including descriptive and thermometer or Visual Analogue Scale (VAS), and serves as a generic validated instrument for use as a measure of HRQL [26]. VAS scores range from 0 (death) to 100 (perfect health).

\section{Statistical analysis \\ Prevalence, health care costs, and resource use}

The estimate for GW prevalence in physician practices was calculated using the number of new or existing GW cases observed, divided by the total number of visiting patients during the 2-week study period. Prevalence was calculated for overall patients, and for each physician specialty type, age group, and sex, reporting also the 95\% CI. Previouslydiagnosed GW patients who sought medical care for other reasons were not considered. A national-level prevalence estimate was extrapolated from the estimated prevalence for each specialty and the distribution of GW patients seeking healthcare among the specialties. The national-level proportion of GW patients who sought care was calculated according to physician specialty, based on the following formula:

$$
G i=(S i * D i) /\left(\sum i=1 \text { to } 4 S i * D i\right)
$$

where $S$ is the number of physicians of a given specialty in the country, $D$ is the mean number of $\mathrm{GW}$ patients seen by the specific specialty (based on 2-week daily logs), and $i$ is the specialty type (PCP, GP/FM, OB/GYN, URO, DERM, IDS). The proportions of patients seeing each specialty type were used in the following formula to derive weights applied to patients included in the study database, and to derive a national-level prevalence estimate:

$$
\mathrm{W}=(\mathrm{Gi} * \mathrm{nT}) /(\mathrm{ni})
$$

where $\mathrm{Gi}$ is the proportion of GW patients at a national level treated within each specialty, $n T$ is the total number of patients included in the study, and $n i$ is total number of patients counted in the study for a given specialty.

Referral patterns reported by physicians were described, reporting the percentage of patients consulted directly by PCP, URO, or DERM as well as those referred by another physician. Healthcare resource use reported by participant physicians in the corresponding survey was described and compared between the physician specialty types. Costs reported in Philippine pesos (PHP) were calculated based on healthcare resource use reported by physicians in the corresponding survey and reported by physician specialty.

\section{Psychosocial impact of GW and selected HPV diseases}

Using the HIP score, survey items were linearly transformed to a $0-100$ scale, with higher scores indicating better conditions. To account for missing data, scale scores were created by computing the mean as the sum of the item scores over the number of items answered. If more than $50 \%$ of the items on the scale were missing, the score was not computed. To create the total scale score, the mean was computed as the sum of all the items over the number of items answered on all scales.

The Japanese version of the EQ-5D instrument was used in this study to estimate the utilities associated with EQ-5D health status [27]. Japan was the first Asian country to develop its own preference weights of EQ-5D since 2002, and the model was chosen to represent Asian preference weights [28]. VAS scores range from 0 (worst HRQL) to 100 (best HRQL), and utility values from 0 (death) to 1 (perfect health).

HIP and EQ-5D scores were compared according to the presence of GW diagnosis (for males) and HPV disease diagnosis (for females). For continuous variables, the Student T-test or Mann-Whitney U-test was performed to compare normal and non-normal variables between subgroups. For categorical variables, Chi-square or Fisher's exact tests were performed depending on the distribution of patients across response categories. CECA scores were also compared, just for patients with GW, by gender and age groups (18-29years, 30-44 years, and 45-60 years) using the Mann-Whitney U-test and Kruskal-Wallis test, respectively.

\section{Results}

Prevalence

A total of 157 physicians (28 PCPs, 43 OB/GYNs, 29 UROs, 29 DERMs, 28 IDSs) participated in the study. The overall prevalence was estimated at $4.78 \%$, with a higher overall prevalence in men (8.00\%) compared with women $(3.39 \%$ (Table 1$)$, data adjusted by specialty). However, overall prevalence decreased to $2.66 \%$ (95\% CI: 2.43-2.89) once adjusted by physician specialty, age, and gender according to the distribution of general population in Philippines.

Regarding GW prevalence rates by patient age, patients aged 50-54 years had the highest rate $(8.20 \%)$, while those aged $>54$ had the lowest (2.69\%) (Fig. 1). Prevalence by gender and physician specialty showed that patients treated by an IDS had a high prevalence among male (19.7\%) and female (17.3\%) patients (Table 1). In addition, prevalence was higher for male patients treated by URO (4.06\%) (Table 1$)$.

For GW patients with physician office visits during the 2-week study period, the percentage of patients with existing GW instead of new GW were higher for OB/GYN practices (40.68\%) followed by PCP (36.36\%), 
Table 1 GW Prevalence in the Philippines by Patient Sex and Physician Specialty

\begin{tabular}{|c|c|c|c|c|c|c|}
\hline & \multicolumn{2}{|l|}{ Male } & \multicolumn{2}{|l|}{ Female } & \multicolumn{2}{|l|}{ Overall } \\
\hline & $\overline{(n / N)}$ & $(\%, 95 \mathrm{Cl})^{2}$ & $\overline{(n / N)}$ & $(\%, 95 \mathrm{Cl})^{2}$ & $\overline{(\mathrm{n} / \mathrm{N})}$ & $(\%, 95 \mathrm{Cl})^{2}$ \\
\hline PCP & $7 / 1590$ & $0.44(0.44 ; 0.44)$ & $4 / 1745$ & $0.23(0.23 ; 0.23)$ & $11 / 3335$ & $0.33(0.33 ; 0.33)$ \\
\hline OB/GYN & $1 / 39$ & $2.56(2.51 ; 2.61)$ & $58 / 2724$ & $2.13(2.12 ; 2.14)$ & $59 / 2763$ & $2.14(2.13 ; 2.14$ \\
\hline URO & $41 / 1009$ & $4.06(4.05 ; 4.08)$ & $0 / 169$ & 0 & $41 / 1178$ & $3.48(3.47 ; 3.49)$ \\
\hline DERM & $21 / 554$ & $3.79(3.78 ; 3.81)$ & $11 / 987$ & $1.11(1.11: 1.12)$ & $32 / 1541$ & $2.08(2.07 ; 2.08)$ \\
\hline IDS & $267 / 1358$ & $19.7(19.64 ; 19.68)$ & $174 / 1004$ & $17.3(17.3 ; 17.4)$ & $441 / 2365$ & $18.7(18.7 ; 18.7)$ \\
\hline OVERALL ${ }^{1}$ & $337 / 4550$ & $8.0(7.69 ; 8.31)$ & $247 / 6629$ & $3.39(3.13 ; 3.65)$ & $584 / 11179$ & $4.78(4.58 ; 4.98)$ \\
\hline
\end{tabular}

${ }^{1}$ Data weighted according to specialty type

${ }^{2}$ Percentage and $95 \% \mathrm{Cl}$ calculated considering the number of patients with identified GW status

Data weighted according to specialty type

Cl confidence interval; DERM dermatologist; GW genital warts; IDS infectious disease specialist; OB/GYN obstetrician/ gynecologist; $P C P$ mprimary care physician, URO urologists

and IDS (33.78\%); and being lower in other practices like DERM (18.75\%) or URO (4.87\%). The distribution of existing cases between recurrent and resistant cases also varied by specialty. The percentage of resistant GW cases ranged from $0 \%$ for PCP and URO consultations to $38.88 \%$ for IDS consultations (Table 2).

\section{Referral patterns, healthcare resource use and costs}

The percentages of male and female patients who consulted directly with that physician without referral were 86.1 and $90.0 \%$ for PCPs, 81.07 and $76.0 \%$ for DERMs, $72.6 \%$ for UROs (males), 93.26\% for OBSGYN (females), and 54.64 and $54.8 \%$ for IDSs (Fig. 2). Following GW diagnosis, the percentages of patients by gender treated by the different physician specialties were $48.5 \%$ (males) and $30.0 \%$ (females) for PCP $76.9 \%$ (males) and $77.4 \%$ (females) for DERM, 98.3\% (males) for URO, 97.3\% (females) for OBGYN, and $81.6 \%$ (males) and $63.0 \%$ (females) for IDS. The mean (SD) number of office visits performed during a GW episode (from initial diagnosis to close of treatment), as reported by participating physicians, was 3.74 (2.29) for males and 3.50 (2.33) for females, both with a range of 1 to 20 visits per episode (minimum and maximum, respectively). The mean number of visits reported was similar across physician specialties, ranging in males from 3.48 for DERM to 4.25 for IDS and in females from 3.00 for PCP to 4.08 for IDS. The number of hospital or ER visits was approximately one-tenth of the number of office visits for the treatment of $1 \mathrm{GW}$ episode. Physicians reported a mean (SD) of 0.21 (1.02) hospital or ER visits per episode in males and $0.3(0.99)$ hospital or ER visits per episode in females (Table 3).

Visual examination was the primary diagnostic technique used by all specialists (Fig. 3). In males, except for biopsy and histological examination, all other tools and techniques (eg, colposcopy, anoscopy-proctoscopy, hybrid capture II-HPV DNA tests) were seldom used to diagnose GW. In females, OB/GYNs used most test

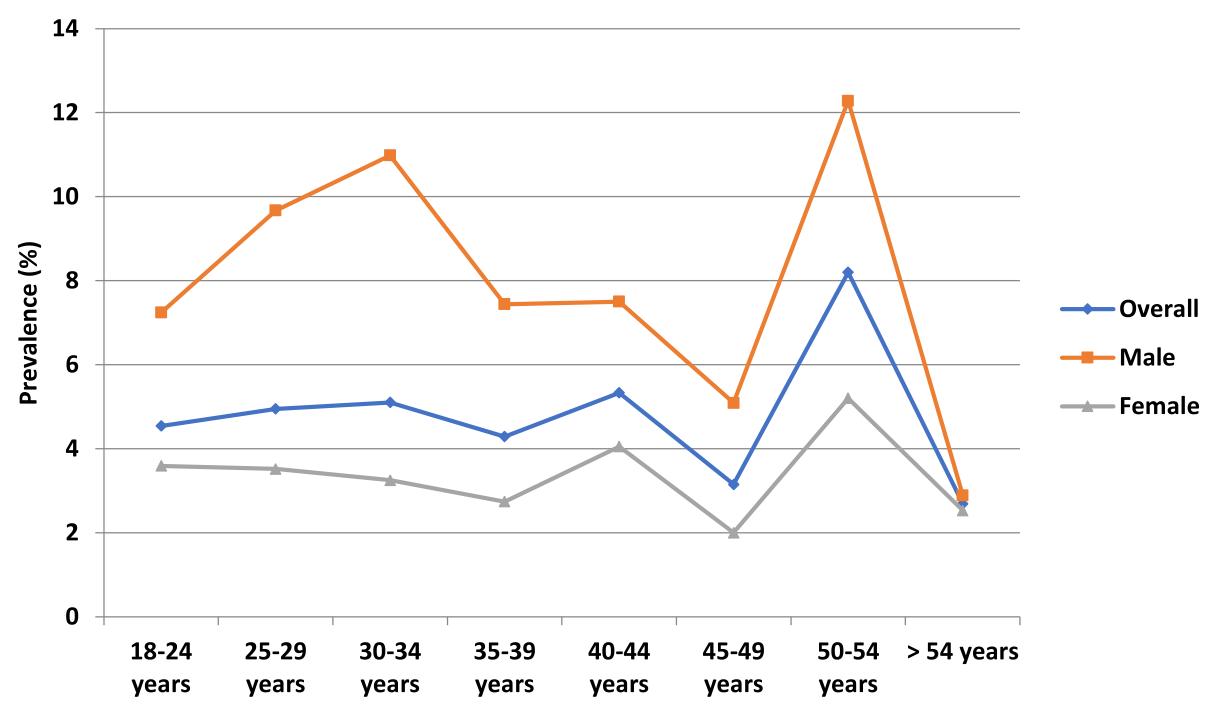

Fig. 1 GW Prevalence by Age and Sex (Weighted). GW = genital warts 
Table 2 Description of GW Cases by Specialty

\begin{tabular}{|c|c|c|c|c|c|}
\hline & $\begin{array}{l}\text { PCP } \\
(n=28)\end{array}$ & DERM $(n=29)$ & $\begin{array}{l}\text { OB/GYN } \\
(n=43)\end{array}$ & $\begin{array}{l}\text { URO } \\
(n=29)\end{array}$ & $\begin{array}{l}\text { IDS } \\
(n=28)\end{array}$ \\
\hline All GW Patients & $11(0.30 \%)$ & $32(2.10 \%)$ & $59(2.10 \%)$ & 41 (3.50\%) & $441(18.50 \%)$ \\
\hline \multicolumn{6}{|c|}{ New or existing GW } \\
\hline New Case & 7 (63.64\%) & $26(81.25 \%)$ & $35(59.32 \%)$ & 39 (95.13\%) & $292(66.22 \%)$ \\
\hline Existing Case & $4(36.36 \%)$ & $6(18.75 \%)$ & $24(40.68 \%)$ & $2(4.87 \%)$ & $149(33.78 \%)$ \\
\hline Valid n & 11 & 32 & 59 & 41 & 441 \\
\hline \multicolumn{6}{|l|}{ Existing cases } \\
\hline Recurrent & $4(100.00 \%)$ & $5(83.33 \%)$ & $20(83.33 \%)$ & $2(100.00 \%)$ & $91(61.12 \%)$ \\
\hline Resistant & $0(0.00 \%)$ & $1(16.67 \%)$ & $4(16.67 \%)$ & $0(0.00 \%)$ & $58(38.88 \%)$ \\
\hline Valid n & 4 & 6 & 24 & 2 & 149 \\
\hline
\end{tabular}

${ }^{1}$ New Case: GW case not diagnosed previously by patient or another physician

${ }^{2}$ Existing Case: GW case was diagnosed previously by patient or another physician

${ }^{3}$ Recurrent Case: GW case where previous episodes were resolved with treatment

${ }^{4}$ Resistant Case: GW case where previous episodes were not resolved with treatment

$D E R M$ dermatologist, GW genital warts, IDS infectious disease specialist, $O B / G Y N$ obstetrician/gynecologist, $P C P$ primary care physician, URO urologists

types, including the Pap smear, more frequently than other specialty physicians. IDS physicians administered visual and histological examinations as well as acetic acid tests more frequently.

In terms of treatments used, electrosurgery was more frequently used by URO, OB/GYN, and DERM physicians; and curettage was utilized more often by DERMs (Fig. 4). In addition to topical medications administered in-office, $\sim 50 \%$ of $\mathrm{GW}$ patients were also prescribed topical medications for at-home use. Imiquimod topical (Aldara ${ }^{\circ}$ ) was more frequently administered by IDSs and DERMs, and it was prescribed more frequently as an at-home rather than an inoffice medication by all specialists (Fig. 5a).

Figure 6 shows the mean costs associated with GW diagnosis and management among male and female patients, overall and by specialty. Female patients incurred higher GW-related costs, as reported by DERMs, followed by OB/GYNs. In male patients, higher costs were reported by UROs, followed by IDS specialists. Healthcare resource utilizations, the overall median and mean costs associated with a GW episode (diagnosis and treatment), were estimated at 7121.30 PHP and 8105.52 PHP (SD: 5823.79), respectively, for men, and 7000.00 PHP and 8786.93 PHP (SD: 9576.08), respectively, for women.

\section{Psychosocial impact of GW and selected HPV diseases}

HIP showed statistically significant differences among male patients with and without GW for all scores, except control/life impact $(p=0.187)$. In all statistically significant scores, males with GW patients reported higher values, reflecting a higher disease impact (scale: 0-100) compared with patients without GW (Table 4). Among female

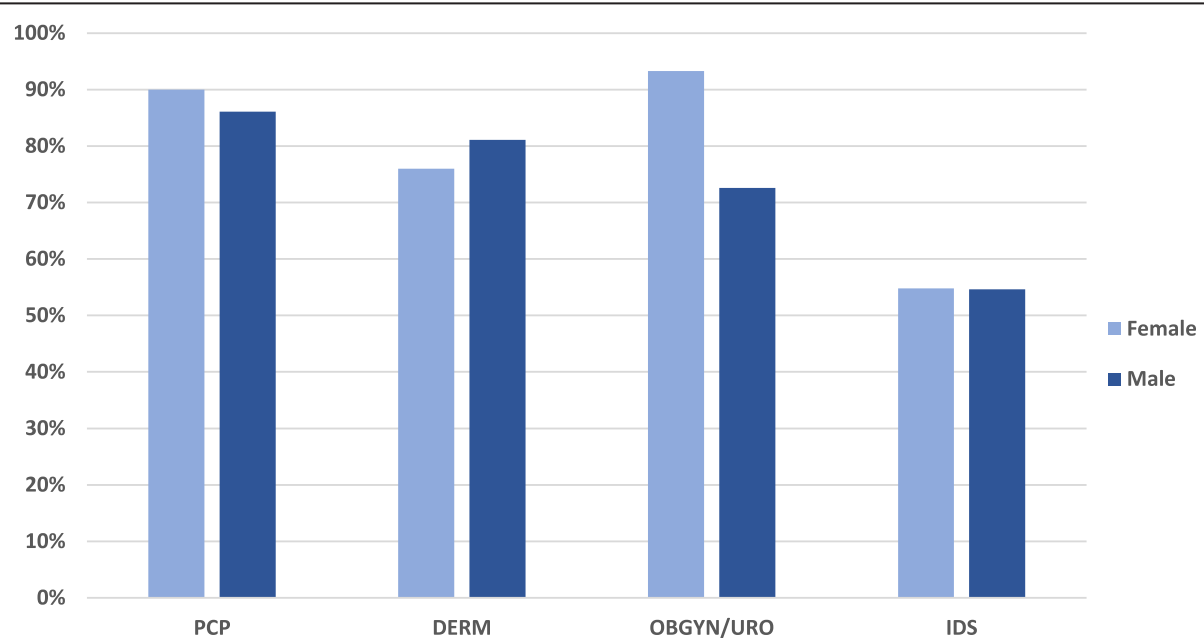

Fig. 2 GW Patients Initially Treated by Consulting Physicians. DERM = dermatologist; GW = genital warts; IDS = infectious disease specialist; $\mathrm{OBGYN}=$ obstetrician/gynecologist; $\mathrm{PCP}=$ primary care physician; $\mathrm{URO}=$ urologist 
Table 3 Health Care Utilizations for GW Patients

\begin{tabular}{|c|c|c|c|c|c|}
\hline & $\begin{array}{l}\text { PCP } \\
(n=28)\end{array}$ & $\begin{array}{l}\text { DERM } \\
(n=29)\end{array}$ & $\begin{array}{l}\text { URO } \\
(n=29)\end{array}$ & $\begin{array}{l}\text { IDS } \\
(n=28)\end{array}$ & $\begin{array}{l}\text { Overall } \\
(n=114)\end{array}$ \\
\hline \multicolumn{6}{|l|}{ Male Patients } \\
\hline \multicolumn{6}{|c|}{ Number of office visits } \\
\hline Mean & 3.50 & 3.48 & 3.59 & 4.25 & 3.74 \\
\hline SD & 1.51 & 1.53 & 1.66 & 3.47 & 2.29 \\
\hline Median & 3.5 & 3.0 & 3.0 & 3.0 & 3.0 \\
\hline Min & 1.0 & 2.0 & 1.0 & 2.0 & 1.0 \\
\hline Max & 6.0 & 7.0 & 8.0 & 20.0 & 20.0 \\
\hline Valid n & 12 & 27 & 29 & 28 & 96 \\
\hline \multicolumn{6}{|c|}{ Number of hospital or ER visits } \\
\hline Mean & 0.00 & 0.72 & 0.08 & 0.00 & 0.21 \\
\hline SD & 0.00 & 1.88 & 0.41 & 0.00 & 1.02 \\
\hline Median & 0.0 & 0.0 & 0.0 & 0.0 & 0.0 \\
\hline Min & 0.0 & 0.0 & 0.0 & 0.0 & 0.0 \\
\hline $\operatorname{Max}$ & 0.0 & 8.0 & 2.0 & 0.0 & 8.0 \\
\hline Valid n & 19 & 25 & 24 & 27 & 95 \\
\hline \multicolumn{6}{|c|}{ Female Patients } \\
\hline & $\begin{array}{c}\text { PCP } \\
(n=28)\end{array}$ & $\begin{array}{c}\text { DERM } \\
(n=29)\end{array}$ & $\begin{array}{c}\mathrm{OB} / \mathrm{GYN} \\
(n=43)\end{array}$ & $\begin{array}{c}\text { IDS } \\
(n=29)\end{array}$ & $\begin{array}{c}\text { Overall } \\
(n=129)\end{array}$ \\
\hline \multicolumn{6}{|c|}{ Number of office visits } \\
\hline Mean & 3.00 & 3.83 & 3.16 & 4.08 & 3.50 \\
\hline SD & 1.71 & 2.60 & 1.17 & 3.57 & 2.33 \\
\hline Median & 2.0 & 3.0 & 3.0 & 3.0 & 3.0 \\
\hline Min & 1.0 & 0.0 & 1.0 & 2.0 & 0.0 \\
\hline Max & 6.0 & 12.0 & 6.0 & 20.0 & 20.0 \\
\hline Valid n & 14 & 24 & 43 & 24 & 105 \\
\hline \multicolumn{6}{|c|}{ Number of hospital or ER visits } \\
\hline Mean & 0.00 & 0.65 & 0.40 & 0.07 & 0.30 \\
\hline SD & 0.00 & 1.58 & 1.04 & 0.38 & 0.99 \\
\hline Median & 0.0 & 0.0 & 0.0 & 0.0 & 0.0 \\
\hline Min & 0.0 & 0.0 & 0.0 & 0.0 & 0.0 \\
\hline Max & 0.0 & 6.0 & 4.0 & 2.0 & 6.0 \\
\hline Valid n & 22 & 23 & 42 & 27 & 114 \\
\hline
\end{tabular}

DERM dermatologist, $E R$ emergency room, GW genital warts, IDS infectious disease specialist, min minimum, max maximum, OB/GYN obstetrician/gynecologist, $P C P$ primary care physician, $S D$ standard deviation, URO urologists

patients, no statistically significant differences were found among those diagnosed with selected HPV diseases in any of the HIP questionnaire domains (Table 4). However, comparisons between study groups showed statistically significant differences between patients with external GW and those with abnormal Pap and HPV positive test results, showing higher disease impact in patients with GW. These differences were identified in the following dimensions: "worries and concerns" $(p=0.018)$, "emotional impact" $(p<0.001)$, "partner issues and transmissions" $(p=0.007)$, and "interaction with doctors" $(p<0.001)$ (Table 8 in
Appendix). Differences were not statistically significant among HPV-related disease groups regarding sexual impact, self-image, and control-life impact.

No significant differences were observed in CECA values for each dimension by gender (CECA mean score; men: 4.19 vs women: $4.10 ; p=0.844$ ) (Table 5) and age groups (CECA mean score; $18-29$ years: 3.91, 30-44 years: 4.12 , and 45-60 years: $4.17 ; p=0.710$ ).

Male patients with GW reported significantly lower mean VAS scores in the EQ-5D (78.2) than those without GW (86.3, $p<0.001$ ) (Table 6); results that reflect the 
(a)

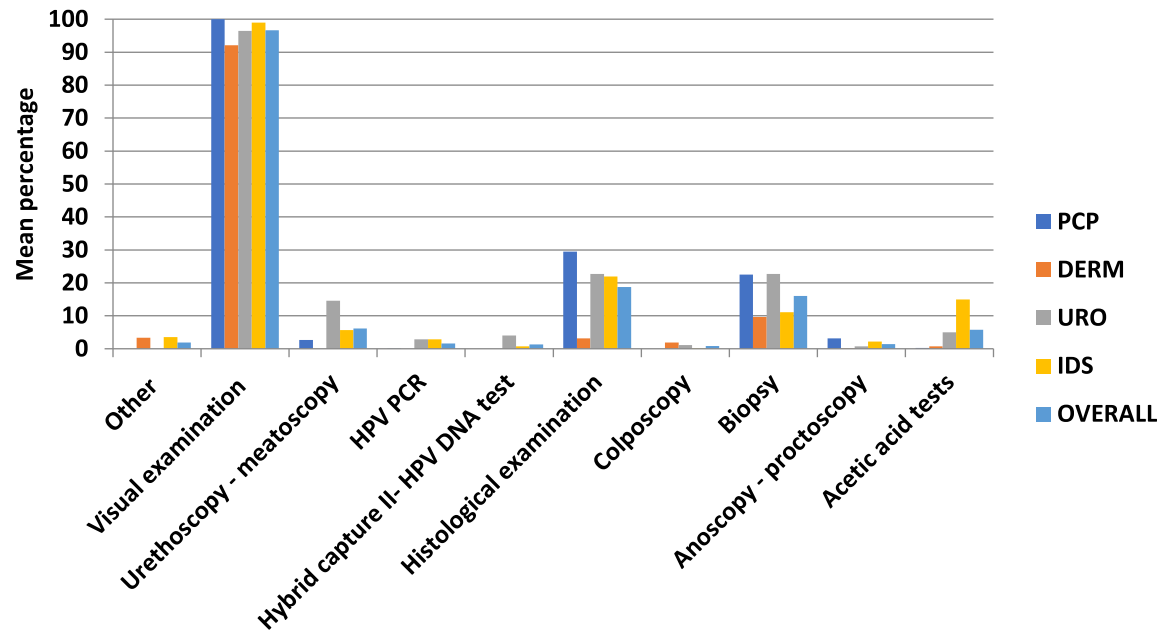

(b)

Last 20 Male Patients

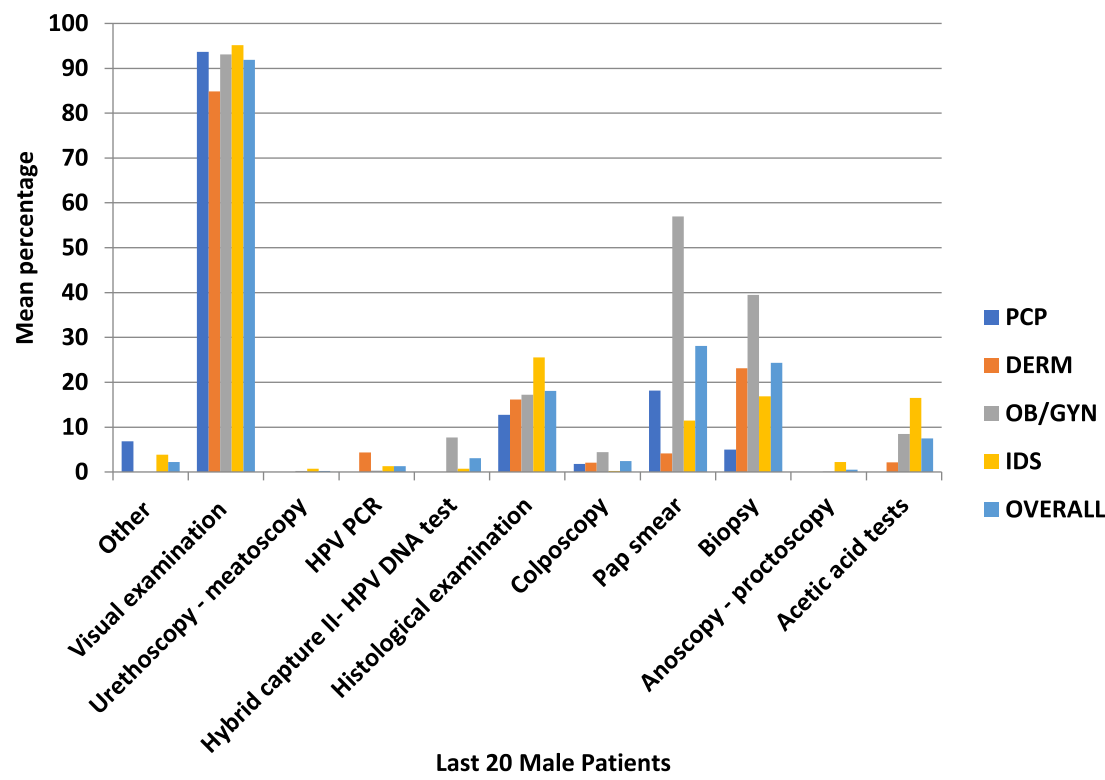

Fig. 3 a GW Diagnostic Tool and Technique Utilization for GW Treatment for Male Patients. DERM = dermatologist; DNA = deoxyribonucleic acid; $\mathrm{GW}=$ genital warts; $\mathrm{HPV}=$ human papillomavirus; IDS = infectious disease specialist; $\mathrm{PCP}=$ primary care physician; $\mathrm{PCR}=$ polymerase chain reaction; URO = urologist. $\mathbf{b}$ GW Diagnostic Tools and Techniques Distribution for Female GW Patients . DERM = dermatologist; DNA = deoxyribonucleic acid; GW = genital warts; HPV = human papillomavirus; IDS = infectious disease specialist; Pap = Papanicolaou test; PCP = primary care physician; $\mathrm{PCR}=$ polymerase chain reaction; $\mathrm{OB} / \mathrm{GYN}=$ obstetrician/ gynecologist

impact of a GW diagnosis on male patients. Utility values were also significantly lower for male GW patients (mean: 0.81) compared with male patients without GW (mean: $0.94, p<0.001)$. In female patients, mean VAS scores (78.0) and utility values (0.84) in patients with selected HPV-related diseases were lower than scores for those without selected HPV-related diseases (78.9 and 0.88, respectively), but the differences were not statistically significant. Like with HIP scores, comparisons between study groups in females showed worse HRQL for patients with
GW than those with abnormal Pap and HPV positive test results (VAS: mean scores of 72.9 vs 80.8 , respectively; utility values: mean scores of 0.79 vs 0.89 , respectively). Differences were statistically significant only for utility values $(p=0.005)$, not for VAS $(0.087)$.

\section{Discussion}

This cross-sectional study estimated the burden of GW in the Philippines by obtaining the GW prevalence rate, 
Table 4 HIP Questionnaire Scores per GW Diagnosis

\begin{tabular}{|c|c|c|c|c|}
\hline Male Patients $(n=149)$ & With GW $(n=74)$ & No GW $(n=75)$ & Overall & $P$-value \\
\hline \multicolumn{5}{|c|}{ HPV Impact Profile Total Score } \\
\hline Mean & 52.35 & 37.72 & 44.99 & \multirow[t]{3}{*}{$<0.0001$} \\
\hline SD & 11.81 & 17.04 & 16.37 & \\
\hline $95 \% \mathrm{Cl}$ & $(49.6 ; 55.1)$ & $(33.8 ; 41.7)$ & $(42.3 ; 47.7)$ & \\
\hline \multicolumn{5}{|l|}{ Worries and Concerns } \\
\hline Mean & 55.85 & 31.38 & 43.62 & \multirow[t]{3}{*}{$<0.0001$} \\
\hline SD & 20.70 & 25.20 & 26.06 & \\
\hline $95 \% \mathrm{Cl}$ & $(51.0 ; 60.7)$ & $(25.5 ; 37.3)$ & $(39.4 ; 47.9)$ & \\
\hline \multicolumn{5}{|l|}{ Emotional Impact } \\
\hline Mean & 48.06 & 31.55 & 39.75 & \multirow[t]{3}{*}{$<0.0001$} \\
\hline SD & 16.22 & 20.56 & 20.25 & \\
\hline $95 \% \mathrm{Cl}$ & $(44.3 ; 51.8)$ & $(26.8 ; 36.3)$ & $(36.4 ; 43.0)$ & \\
\hline \multicolumn{5}{|l|}{ Sexual Impact } \\
\hline Mean & 49.45 & 43.11 & 46.26 & \multirow[t]{3}{*}{0.0199} \\
\hline SD & 14.71 & 17.80 & 16.59 & \\
\hline $95 \% \mathrm{Cl}$ & $(46.0 ; 52.9)$ & $(39.0 ; 47.2)$ & $(43.6 ; 49.0)$ & \\
\hline \multicolumn{5}{|l|}{ Self-image } \\
\hline Mean & 46.27 & 40.74 & 43.49 & \multirow[t]{3}{*}{0.0371} \\
\hline SD & 14.80 & 16.94 & 16.10 & \\
\hline $95 \% \mathrm{Cl}$ & $(42.8 ; 49.7)$ & $(36.8 ; 44.7)$ & $(40.9 ; 46.1)$ & \\
\hline \multicolumn{5}{|c|}{ Partner Issues and Transmission } \\
\hline Mean & 60.89 & 48.09 & 54.63 & \multirow[t]{3}{*}{0.0008} \\
\hline SD & 15.97 & 26.90 & 22.84 & \\
\hline $95 \% \mathrm{Cl}$ & $(57.1 ; 64.7)$ & $(41.6 ; 54.6)$ & $(50.8 ; 58.5)$ & \\
\hline \multicolumn{5}{|l|}{ Interactions with Doctors } \\
\hline Mean & 55.38 & 35.85 & 46.61 & \multirow[t]{3}{*}{$<0.0001$} \\
\hline SD & 17.08 & 22.41 & 21.87 & \\
\hline $95 \% \mathrm{Cl}$ & $(51.3 ; 59.5)$ & $(29.9 ; 41.8)$ & $(42.8 ; 50.5)$ & \\
\hline \multicolumn{5}{|l|}{ Control/Life Impact } \\
\hline Mean & 50.41 & 46.49 & 48.44 & \multirow[t]{3}{*}{0.1868} \\
\hline SD & 13.98 & 21.12 & 17.98 & \\
\hline $95 \% \mathrm{Cl}$ & $(47.1 ; 53.7)$ & $(41.6 ; 51.4)$ & $(45.5 ; 51.4)$ & \\
\hline Female Patients $(n=225)$ & HPV disease $(n=182)$ & No HPV disease $(n=43)$ & Overall & $P$-value \\
\hline \multicolumn{5}{|c|}{ HPV Impact Profile Total Score } \\
\hline Mean & 47.98 & 48.06 & 47.99 & \multirow[t]{3}{*}{0.9800} \\
\hline SD & 19.85 & 20.56 & 19.94 & \\
\hline $95 \% \mathrm{Cl}$ & $(45.1 ; 50.9)$ & $(41.7 ; 54.5)$ & $(45.4 ; 50.6)$ & \\
\hline \multicolumn{5}{|l|}{ Worries and Concerns } \\
\hline Mean & 49.56 & 49.11 & 49.47 & \multirow[t]{3}{*}{0.9252} \\
\hline SD & 27.11 & 30.74 & 27.75 & \\
\hline $95 \% \mathrm{Cl}$ & $(45.6 ; 53.5)$ & $(39.5 ; 58.7)$ & $(45.8 ; 53.1)$ & \\
\hline \multicolumn{5}{|l|}{ Emotional Impact } \\
\hline Mean & 46.72 & 45.62 & 46.52 & \multirow[t]{3}{*}{0.7943} \\
\hline SD & 24.68 & 24.71 & 24.64 & \\
\hline $95 \% \mathrm{Cl}$ & $(43.1 ; 50.3)$ & $(37.9 ; 53.3)$ & $(43.3 ; 49.8)$ & \\
\hline
\end{tabular}


Table 4 HIP Questionnaire Scores per GW Diagnosis (Continued)

\begin{tabular}{|c|c|c|c|c|}
\hline Male Patients $(n=149)$ & With GW $(n=74)$ & No GW $(n=75)$ & Overall & $P$-value \\
\hline \multicolumn{5}{|l|}{ Sexual Impact } \\
\hline Mean & 49.02 & 51.58 & 49.47 & \multirow[t]{3}{*}{0.5165} \\
\hline SD & 22.51 & 19.77 & 22.03 & \\
\hline $95 \% \mathrm{Cl}$ & $(45.7 ; 52.3)$ & $(45.1 ; 58.1)$ & $(46.5 ; 52.4)$ & \\
\hline \multicolumn{5}{|l|}{ Self-image } \\
\hline Mean & 43.59 & 44.31 & 43.72 & \multirow[t]{3}{*}{0.8314} \\
\hline SD & 19.37 & 21.09 & 19.66 & \\
\hline $95 \% \mathrm{Cl}$ & $(40.7 ; 46.4)$ & $(37.7 ; 50.9)$ & $(41.1 ; 46.3)$ & \\
\hline \multicolumn{5}{|l|}{ Partner Issues and Transmission } \\
\hline Mean & 55.28 & 54.91 & 55.22 & \multirow[t]{3}{*}{0.9375} \\
\hline SD & 26.02 & 27.37 & 26.19 & \\
\hline $95 \% \mathrm{Cl}$ & $(51.4 ; 59.2)$ & $(45.6 ; 64.2)$ & $(51.7 ; 58.8)$ & \\
\hline \multicolumn{5}{|l|}{ Interactions with Doctors } \\
\hline Mean & 42.72 & 41.30 & 42.46 & \multirow[t]{3}{*}{0.7265} \\
\hline SD & 23.76 & 22.05 & 23.41 & \\
\hline $95 \% \mathrm{Cl}$ & $(39.2 ; 46.2)$ & $(34.3 ; 48.3)$ & $(39.4 ; 45.6)$ & \\
\hline \multicolumn{5}{|l|}{ Control/Life Impact } \\
\hline Mean & 49.83 & 54.88 & 50.77 & \multirow[t]{3}{*}{0.1134} \\
\hline SD & 18.94 & 16.88 & 18.64 & \\
\hline $95 \% \mathrm{Cl}$ & $(47.1 ; 52.6)$ & $(49.6 ; 60.1)$ & $(48.3 ; 53.2)$ & \\
\hline \multicolumn{5}{|c|}{ HPV Impact Profile Total Score Categorized } \\
\hline No or little impact & $64(35.2 \%)$ & $15(34.9 \%)$ & $79(35.1 \%)$ & \multirow[t]{4}{*}{0.9983} \\
\hline Moderate impact & $88(48.4 \%)$ & $21(48.8 \%)$ & 109 (48.4\%) & \\
\hline Heavy psychological impact & $30(16.5 \%)$ & $7(16.3 \%)$ & $37(16.4 \%)$ & \\
\hline Valid $n$ & 182 & 43 & 225 & \\
\hline
\end{tabular}

HPV human papillomavirus, $H I P=H P V$ impact profile, $C l$ confidence interval, $G W$ genital warts, $S D$ standard deviation

Table 5 CECA Questionnaire Scores from GW Patients

\begin{tabular}{lllll}
\hline $\begin{array}{l}\text { Patients with GW } \\
(n=121)\end{array}$ & $\begin{array}{l}\text { Male } \\
(n=74)\end{array}$ & $\begin{array}{l}\text { Female } \\
(n=47)\end{array}$ & Overall & P-value \\
\hline \multicolumn{5}{c}{ Emotional health } \\
Mean & 6.51 & 6.63 & 6.56 & 0.8692 \\
SD & 3.75 & 4.65 & 4.11 & \\
$95 \%$ Cl & $(5.6 ; 7.4)$ & $(5.3 ; 8.0)$ & $(5.8 ; 7.3)$ & \\
Sexual activity & & & & \\
Mean & 11.54 & 10.64 & 11.18 & 0.4637 \\
SD & 6.16 & 7.08 & 6.52 & \\
$95 \%$ Cl & $(10.1 ; 13.0)$ & $(8.6 ; 12.7)$ & $(10.0 ; 12.4)$ & \\
CECA total score & & & 0.8437 \\
Mean & 4.19 & 4.10 & 4.15 & \\
SD & 2.13 & 2.60 & 2.32 & $(3.7 ; 4.6)$ \\
95\% Cl & $(3.7 ; 4.7)$ & $(3.3 ; 4.9)$ & \\
\hline
\end{tabular}

CECA Cuestionario Específico para Condiloma Acuminado (Specific questionnaire for Condylomata Acuminata), Cl confidence interval, GW genital warts, SD standard deviation
GW-related resource utilization and costs, and data on the self-reported HPV disease-related psychosocial impact among male and female patients aged 18-60 years.

At the national level, the current study estimated GW prevalence at $8.00 \%$ among men and $3.39 \%$ among women, which is higher than figures reported in currently available literature $[7,29,30]$. However, overall prevalence decreased to $2.66 \%$ once adjusted by age, gender, and physician specialty. A previous study performed in Hong Kong, including 170 private doctors working in Social Hygiene Clinics and using the same study approach, estimated an overall GW prevalence rate of $0.94 \%$ [15] - significantly lower than this study's findings on the Philippines.

Recent studies in Europe, the United States, and Australia are focused primarily on estimating $\mathrm{GW}$ incidence rather than prevalence. However, the results of a 2007 study of women aged 18-45 years in 4 northern European countries over a 12-month period showed a GW prevalence of $1.3 \%$ in Denmark, $1.9 \%$ in Iceland, $1.1 \%$ in Norway, and $1.0 \%$ in Sweden [31]. A recent study performed in Peru using the same methodology reported also similar prevalence rates [32]. 
Table 6 EQ-5D, VAS Score and Utility: Patients with and without GW

\begin{tabular}{|c|c|c|c|c|}
\hline Male Patients $(n=149)$ & $\begin{array}{l}\text { With GW } \\
(n=74)\end{array}$ & No GW $(n=75)$ & Overall & $P$-value \\
\hline \multicolumn{5}{|l|}{ Mobility } \\
\hline I have no problems in walking about. & $64(88.9 \%)$ & $67(93.1 \%)$ & 131 (91.0\%) & \multirow[t]{2}{*}{0.3830} \\
\hline I have some problems in walking about. & $8(11.1 \%)$ & $5(6.9 \%)$ & $13(9.0 \%)$ & \\
\hline \multicolumn{5}{|l|}{ Self-Care } \\
\hline I have no problems with self-care. & $66(90.4 \%)$ & $71(98.6 \%)$ & 137 (94.5\%) & \multirow[t]{2}{*}{0.0306} \\
\hline $\begin{array}{l}\text { I have some problems washing or dressing } \\
\text { myself. }\end{array}$ & $7(9.6 \%)$ & $1(1.4 \%)$ & $8(5.5 \%)$ & \\
\hline \multicolumn{5}{|l|}{ Usual Activities } \\
\hline $\begin{array}{l}\text { I have no problems with performing my usual } \\
\text { activities. }\end{array}$ & $62(84.9 \%)$ & $69(95.8 \%)$ & $131(90.3 \%)$ & \multirow[t]{2}{*}{0.0263} \\
\hline $\begin{array}{l}\text { I have some problems with performing my } \\
\text { usual activities. }\end{array}$ & $11(15.1 \%)$ & $3(4.2 \%)$ & $14(9.7 \%)$ & \\
\hline \multicolumn{5}{|l|}{ Pain-Discomfort } \\
\hline I have no pain or discomfort. & $43(58.9 \%)$ & $60(85.7 \%)$ & $103(72.0 \%)$ & \multirow[t]{3}{*}{0.0015} \\
\hline I have moderate pain or discomfort. & $29(39.7 \%)$ & $10(14.3 \%)$ & $39(27.3 \%)$ & \\
\hline I have extreme pain or discomfort. & $1(1.4 \%)$ & & $1(0.7 \%)$ & \\
\hline \multicolumn{5}{|l|}{ Anxiety-Depression } \\
\hline I am not anxious or depressed. & $30(41.7 \%)$ & $63(90.0 \%)$ & $93(65.5 \%)$ & \multirow[t]{3}{*}{$<0.0001$} \\
\hline I am moderately anxious or depressed. & $40(55.6 \%)$ & $6(8.6 \%)$ & $46(32.4 \%)$ & \\
\hline I am extremely anxious or depressed. & $2(2.8 \%)$ & $1(1.4 \%)$ & $3(2.1 \%)$ & \\
\hline \multicolumn{5}{|l|}{ VAS (EQ-5D) } \\
\hline Mean & 78.20 & 86.34 & 82.33 & \multirow[t]{3}{*}{$<0.0001$} \\
\hline SD & 13.63 & 10.62 & 12.82 & \\
\hline $95 \% \mathrm{Cl}$ & $(75.0 ; 81.4)$ & $(83.9 ; 88.8)$ & $(80.2 ; 84.4)$ & \\
\hline \multicolumn{5}{|l|}{ Utility Values } \\
\hline Mean & 0.81 & 0.94 & 0.87 & \multirow[t]{3}{*}{$<0.0001$} \\
\hline SD & 0.16 & 0.12 & 0.15 & \\
\hline $95 \% \mathrm{Cl}$ & $(0.8 ; 0.8)$ & $(0.9 ; 1.0)$ & $(0.8 ; 0.9)$ & \\
\hline Female Patients $(n=225)$ & $\begin{array}{c}\text { HPV } \\
(n=182)\end{array}$ & No HPV $(n=43)$ & Overall & $P$-value \\
\hline \multicolumn{5}{|l|}{ Mobility } \\
\hline I have no problems in walking about. & $163(90.1 \%)$ & $39(90.7 \%)$ & $202(90.2 \%)$ & \multirow[t]{2}{*}{0.8987} \\
\hline I have some problems in walking about. & $18(9.9 \%)$ & $4(9.3 \%)$ & $22(9.8 \%)$ & \\
\hline \multicolumn{5}{|l|}{ Self-Care } \\
\hline I have no problems with self-care. & $172(96.6 \%)$ & $42(97.7 \%)$ & $214(96.8 \%)$ & \multirow[t]{2}{*}{0.7254} \\
\hline $\begin{array}{l}\text { I have some problems washing or dressing } \\
\text { myself. }\end{array}$ & $6(3.4 \%)$ & $1(2.3 \%)$ & $7(3.2 \%)$ & \\
\hline \multicolumn{5}{|l|}{ Usual Activities } \\
\hline $\begin{array}{l}\text { I have no problems with performing my } \\
\text { usual activities. }\end{array}$ & $158(87.3 \%)$ & $39(90.7 \%)$ & 197 (87.9\%) & \multirow[t]{3}{*}{0.7684} \\
\hline $\begin{array}{l}\text { I have some problems with performing my } \\
\text { usual activities. }\end{array}$ & $22(12.2 \%)$ & $4(9.3 \%)$ & $26(11.6 \%)$ & \\
\hline I am unable to perform my usual activities. & $1(0.6 \%)$ & & $1(0.4 \%)$ & \\
\hline \multicolumn{5}{|l|}{ Pain-Discomfort } \\
\hline I have no pain or discomfort. & $124(68.1 \%)$ & $35(81.4 \%)$ & 159 (70.7\%) & \multirow[t]{2}{*}{0.2178} \\
\hline I have moderate pain or discomfort. & $57(31.3 \%)$ & $8(18.6 \%)$ & $65(28.9 \%)$ & \\
\hline
\end{tabular}


Table 6 EQ-5D, VAS Score and Utility: Patients with and without GW (Continued)

\begin{tabular}{|c|c|c|c|c|}
\hline I have extreme pain or discomfort. & $1(0.5 \%)$ & & $1(0.4 \%)$ & \\
\hline \multicolumn{5}{|l|}{ Anxiety-Depression } \\
\hline I am not anxious or depressed. & $88(48.6 \%)$ & $26(60.5 \%)$ & $114(50.9 \%)$ & \multirow[t]{3}{*}{0.3770} \\
\hline I am moderately anxious or depressed. & $82(45.3 \%)$ & $15(34.9 \%)$ & $97(43.3 \%)$ & \\
\hline I am extremely anxious or depressed. & $11(6.1 \%)$ & $2(4.7 \%)$ & $13(5.8 \%)$ & \\
\hline \multicolumn{5}{|l|}{ VAS (EQ-5D) } \\
\hline Mean & 77.98 & 78.93 & 78.17 & \multirow[t]{3}{*}{0.7121} \\
\hline SD & 14.89 & 15.91 & 15.06 & \\
\hline $95 \% \mathrm{Cl}$ & $(75.8 ; 80.2)$ & $(74.0 ; 83.8)$ & $(76.2 ; 80.2)$ & \\
\hline \multicolumn{5}{|l|}{ Utility Values } \\
\hline Mean & 0.84 & 0.88 & 0.84 & \multirow[t]{3}{*}{0.1010} \\
\hline SD & 0.15 & 0.14 & 0.15 & \\
\hline $95 \% \mathrm{Cl}$ & $(0.8 ; 0.9)$ & $(0.8 ; 0.9)$ & $(0.8 ; 0.9)$ & \\
\hline
\end{tabular}

Cl confidence interval, EQ-5D EuroQol-5 dimension, GW genital warts, HPV human papillomavirus, SD standard deviation, VAS visual analogue scale

The difference in the prevalence of GW between the European studies and this study on the Philippines may be related to sexual behavior differences or differing methods in collecting GW patient data. In the European studies, the median number of sexual partners during a patient's lifetime was 5 , and the median age at the time of first intercourse was 16 years [31], compared to medians of 2 sexual partners and an age of 20 years in the Philippines. However, the median number of sexual partners in the Philippines study accounted only for the most recent 5 years, not a patient's entire lifetime, as reported in the European study. Differences in terms of age at first diagnosis may also be related to the methods used to collect patient data [33].

Considering age distribution, GW diagnoses differed by gender. In this study, the oldest (aged 50-54 years) female patients showed the highest prevalence. Since most HPV infections occurred soon after sexual debut and are transient, women who are over age 30 and HPV positive include persistent carriers and those with new infections. While most studies show a decrease in HPV prevalence with age, many studies conducted in several different international regions have indicated a peak prevalence of HPV infection in women below age 25 [12], a decrease among women aged 35-54, and a second HPV prevalence peak after age 55 [34].

The study found that GW prevalence was higher in the Philippines among men than for women for all age groups. Nevertheless, a notably higher prevalence was recorded for all patients aged 50-54 years. The prevalence tendency exhibited by male patients according to age, excluding those aged 50-54 years, was similar to the prevalence reported in a Korean study examining the epidemiological characteristics of genital herpes and condyloma acuminata among patients presenting to URO and OB/GYN clinics. Analysis by age group and physician specialty showed a high prevalence among male patients treated by an IDS. It is possible these patients have selfselected their specialist; the referral pattern may show a selection of those thought to have the better knowledge of GW management. The predominant age group of patients with condyloma acuminata was 25-29 years in Korean male patients, with a prevalence of $1.84 \%$ [35]. A separate retrospective study of male patients attending a sexually transmitted infection clinic in India reported that $59.7 \%$ of sexually transmitted disease cases occurred among patients aged 25-44 years [29].

Most GW patients included in this study were newly diagnosed cases (66.06\%). Among those with existing GW, $67.48 \%$ were recurrent and $35.52 \%$ were resistant. There is currently no previously published data related to the proportion of new or existing GW cases in the Philippines. However, compared with a previous study using similar methodology but performed in Hong Kong [15], a different distribution was observed; approximately one-tenth (73) of the 721 episodes were designated as new cases. The differences in results may suggest that patients in the Philippines have access to better treatment, and therefore cases are less resistant and recurrent. However, it is possible that lesions presented may have not been noted by the physician, leading to a lower proportion of patients with existing GW (33.94\%) being reported compared to that observed in Hong Kong. Results obtained in terms of healthcare resource use are aligned with other countries in which the objectives of the current study have been also assessed using the same methodology [36, 37].

Diagnosis and treatment of GW was associated with a median cost of 7121 PHP ( $\$ 134$ USD) for men, and 7000 PHP ( $\sim 132$ USD) for women, which is about $8.58 \%$ of the average GDP per capita in the Philippines from 1960 to 2016 [38]. It also equals about $3.63 \%$ of 2012 annual family expenditures in the Philippines [39]. In-office 
(a)

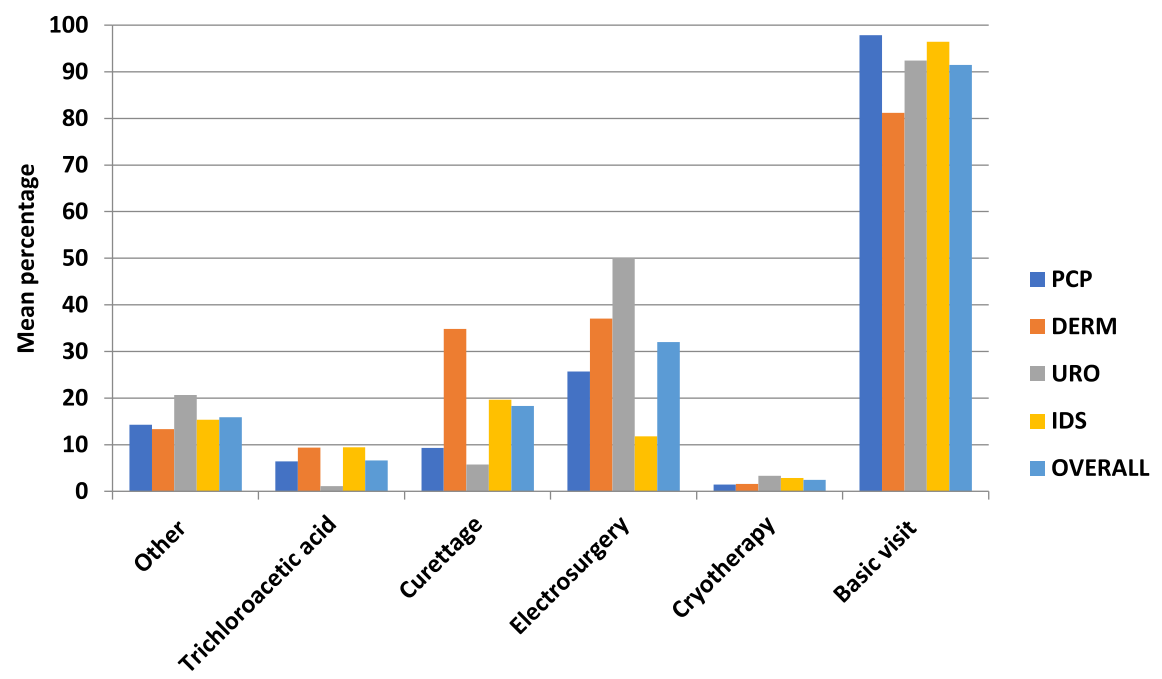

(b)

Last 20 Male Patients

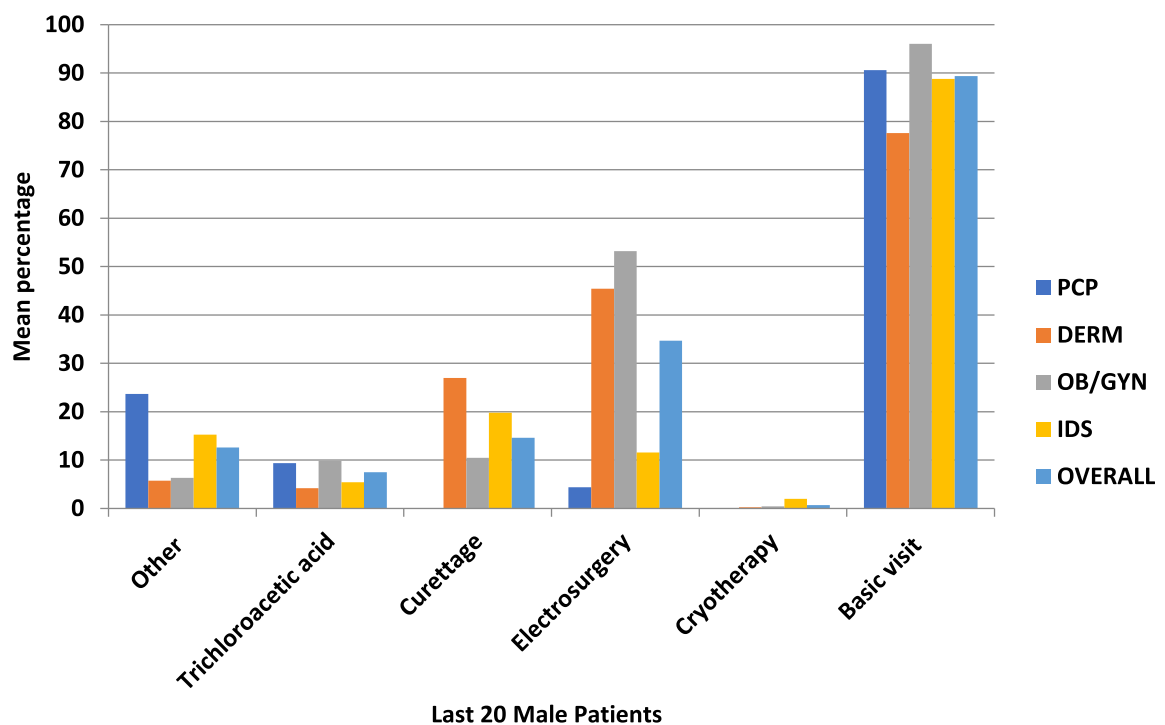

Fig. 4 a In-office Treatment/Procedures for Male GW Patients. DERM = dermatologist; GW = genital warts; IDS = infectious disease specialist; PCP = primary care physician; URO = urologist. $\mathbf{b}$ In-office Treatment/Procedures for Female GW Patients. DERM = dermatologist; GW = genital warts; IDS = infectious disease specialist; OB/GYN = obstetrician/ gynecologist; $\mathrm{PCP}=$ primary care physician

treatment and procedures $(50.46 \%$ of the total) were the highest overall cost drivers for male participants, followed by office visits (24.74\%), and diagnostic tools and techniques $(12.60 \%)$. For female participants, cost drivers were in-office treatments and procedures (53.38\%), followed by office visits (20.02\%), and diagnostic tools and techniques (15.85\%). Costs associated with GW have been assessed in several other countries, including the United States, Canada, and Australia, but the socioeconomic situations and healthcare systems in these countries are not comparable to those in the Philippines.
When analyzing the psychosocial impact of GW on men, those with GW reported worse HRQL scores than those without. Despite the significant differences (particularly among men) in the results of the global HIP scores and EQ$5 \mathrm{D}$ questionnaires, scores were similar for those with and without the disease, reflecting a lack of psychological impact in these areas. In the disease-specific HIP questionnaire, GW patients reported worse HRQL scores compared to those without GW. On HRQL, female GW patients reported a higher psychosocial impact of the disease than male GW patients. The results align with differences reported in previous studies in other disease areas [40-42]. 
(a)

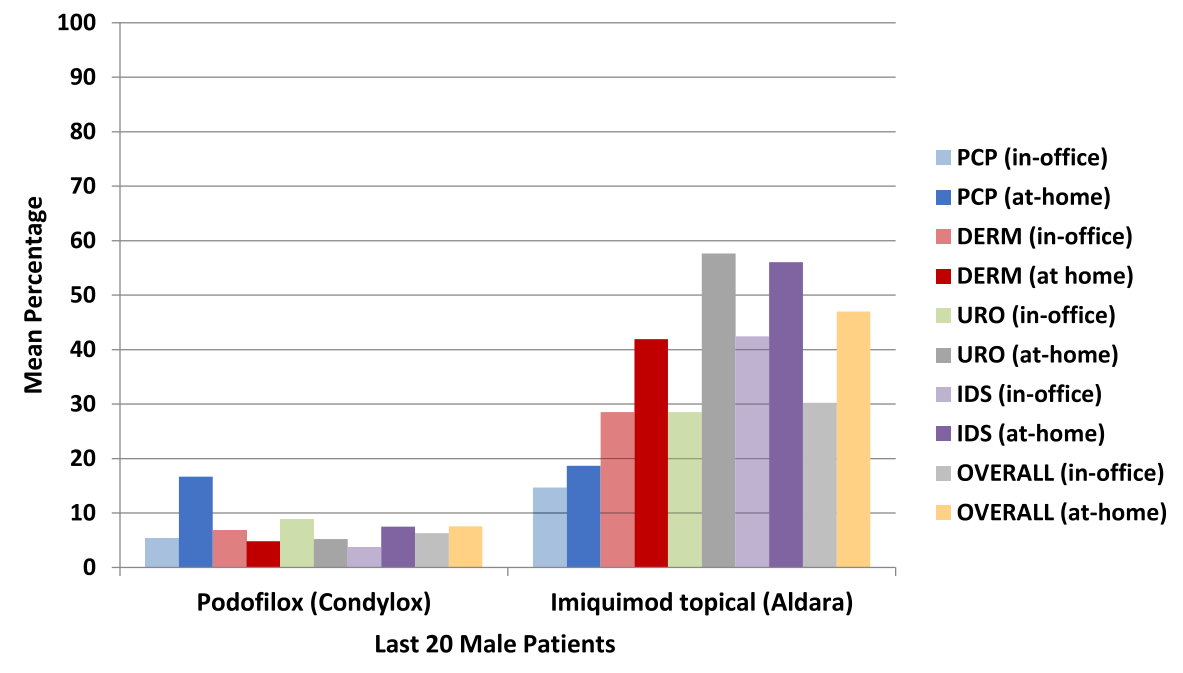

(b)

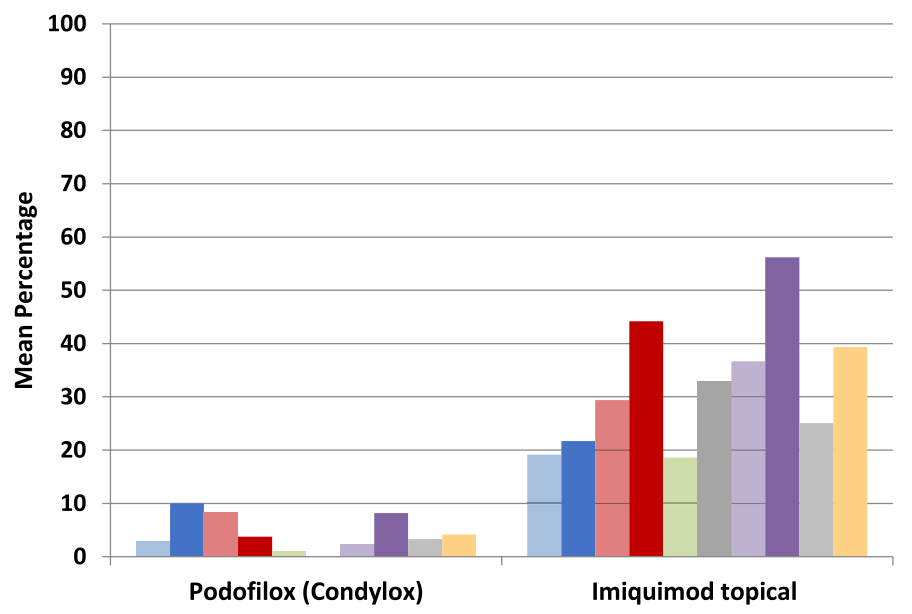

PCP (in-office)

- PCP (at-home)

DERM (in-office)

- DERM (at home)

OB/GYN (in-office)

OB/GYN (at-home)

$\square$ IDS (in-office)

IDS (at-home)

- OVERALL (in-office)

OVERALL (at-home)

Last 20 Male Patients

Fig. 5 a Male GW Patients Prescribed In-office and At-home Topical Medication. DERM = dermatologist; GW = genital warts; IDS = infectious disease specialist; $P C P$ = primary care physician; URO = urologist. b Female GW Patients Prescribed In-office and At-home Topical Medication. DERM = dermatologist; GW = genital warts; IDS = infectious disease specialist; OB/GYN = obstetrician/ gynecologist; PCP = primary care physician

When comparing female HPV-related disease subgroups, results showed a higher psychological impact on female GW patients than on those with other HPV diseases, including precancerous lesions and CIN2/3. In the HIP questionnaire, GW patients reported experiencing a major impact, especially related to "worries and concerns," "emotional impact," "partner issues and transmissions," and "interactions with doctors." The higher disease burden on patients may be associated with the presence of physical lesions visual to the patient, similar to a previous study that examined GW burden [12]. In a recently published study conducted in India including HIV, HPV, and herpes patients, World Health Organization Quality of Life-BREF scores for patients in different STI groups were significantly lower than for those without these conditions [43]. In South Korea, a recent study analyzed questions posted by patients on an HPV informational website [44] and concluded that, based on the type of questions posted, GW can be considered an embarrassing topic for patients.

The current study detected a lower impact on patients with GW (assessed by EQ-5D) than previous studies reported. According to Sénécal et al [45]. in a study in Canada, GW was associated with lower HRQL scores for "pain-discomfort," "anxiety-depression," and 


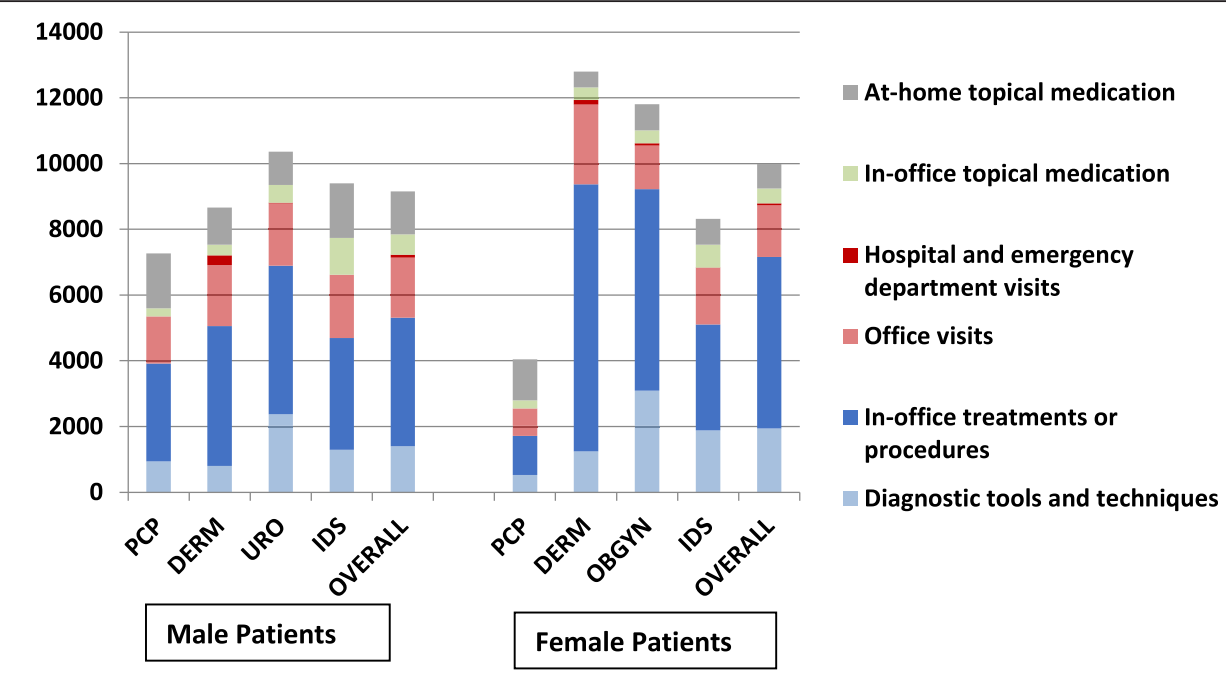

Fig. 6 Mean Costs Associated with GW Diagnosis and Treatment (Philippine Pesos). DERM = dermatologist; GW = genital warts; IDS = infectious disease specialist; $\mathrm{PCP}=$ primary care physician; $\mathrm{OB} / \mathrm{GYN}=$ obstetrician/gynecologist; $\mathrm{URO}=$ urologist

"usual activities." The same Canadian study also showed an absolute difference in EQ-5D utility scores and EQ-VAS health status between GW patients and the population norms of 9.9 (95\% CI: 7.3 to 12.5 ) and 6.0 (95\% CI: 4.1 to 7.9 ) percentage points, respectively. In the current study, the differences in mean VAS and EQ-5D utility scores between patients with and without GW (and other selected HPV-related diseases in female patients) were 8.1 and 0.1 in men, and 0.95 and 0.04 in women, respectively.

The study results are consistent with previously reported results by Wang et al., in China [46]. According to this study, female GW patients had the highest mean HIP scores (52.2), showing a high psychological impact, followed by:

- patients with precancerous cervical lesions (48.6);

- HPV-positive after abnormal Pap (45.8);

- abnormal Pap test results without an HPV test (44.1);

- HPV diagnosis after abnormal Pap results (43.1); and

- normal Pap results (33.1).

A similar study performed by Wang et al. in Taiwan reported a significant psychological impact on women diagnosed with abnormal Pap results, CIN, positive high-risk HPV test, and GW, compared with women with normal Pap test results. Female patients diagnosed with GW showed the highest psychological impact scores [47].

\section{Limitations}

Only physicians treating a higher number of GW patients were included in the study, which may have led to an overestimation of GW when the resulting data was projected to the national level. GW patients who did not seek treatment were not included in the study, which may have underestimated the true prevalence in the Philippines, since weighting was applied. Moreover, overestimation of prevalence may have occurred as a high percentage of GW patients treated by IDSs were referred by other physicians. Despite the specific question about referrals and the exclusion of referred patients, some patients initially diagnosed by other specialists and then referred to IDSs for management may have been double-counted.

Potential bias related to the study information source may exist, as the recall of healthcare resource utilization estimated by physicians may be difficult to control. Additionally, the current study cannot determine whether the psychosocial impact on study participants drawn from the 4 Philippine cities is generalizable to the entire population.

\section{Conclusions}

With an age, gender, and specialty weighted overall prevalence of $2.66 \%, \mathrm{GW}$ poses a significant healthcare and economic concern in the Philippines. Median costs are estimated at 7121 PHP for men, and 7000 PHP for women ( $\$ 134-\$ 132$ USD), which is about $8.58 \%$ of the country's average GDP per capita over the past two decades. In terms of HRQL, the study results suggest that a GW diagnosis in men and women has a negative impact on patient well-being and HRQL. For women, a greater impact was observed among GW patients compared with those with other selected HPV-related diseases. The baseline data in this study offers a foundation for measuring GW prevalence and its psychosocial impact on the overall population. Despite its limitations, this study provides an estimation of GW-related data in the Philippines not previously available. 


\section{Appendix}

Table 7 Participating Physicians by Region

\begin{tabular}{|c|c|c|c|c|c|c|}
\hline$q$ & $\begin{array}{l}\text { PCP } \\
(n=28)\end{array}$ & $\begin{array}{l}\text { DERM } \\
(n=29)\end{array}$ & $\begin{array}{l}\text { OB/GYN } \\
(n=43)\end{array}$ & $\begin{array}{l}\text { URO } \\
(n=29)\end{array}$ & $\begin{array}{l}\text { IDS } \\
(n=28)\end{array}$ & $\begin{array}{l}\text { Overall } \\
(n=157)\end{array}$ \\
\hline \multicolumn{7}{|l|}{ Region } \\
\hline Manila & 22 (91.7\%) & 17 (68.0\%) & $23(54.8 \%)$ & 9 (42.9\%) & 15 (83.3\%) & $86(66.2 \%)$ \\
\hline Luzon & $2(8.3 \%)$ & $3(12.0 \%)$ & 11 (26.2\%) & 4 (19.0\%) & & $20(15.4 \%)$ \\
\hline Mindanao & & $5(20.0 \%)$ & $4(9.5 \%)$ & $5(23.8 \%)$ & 2 (11.1\%) & $16(12.3 \%)$ \\
\hline Visayas & & & $4(9.5 \%)$ & $3(14.3 \%)$ & $1(5.6 \%)$ & 8 (6.2\%) \\
\hline Valid n & 24 & 25 & 42 & 21 & 18 & 130 \\
\hline
\end{tabular}

Percentages calculated over the corresponding valid $\mathrm{n}$

DERM dermatologist, IDS infectious disease specialist, OB/GYN obstetrician/ gynecologist, PCP primary care physician, URO urologist 
Table 8 HIP Questionnaire Scores by HPV-related Disease for Female Patients

\begin{tabular}{|c|c|c|c|c|c|c|c|}
\hline \multicolumn{8}{|l|}{ HPV Disease $(n=182)$} \\
\hline & $\begin{array}{l}\text { Abnormal PAP } \\
(n=46)\end{array}$ & $\begin{array}{l}\text { Abnormal Pap/HPV+ } \\
(n=45)\end{array}$ & $\begin{array}{l}\text { Precancerous } \\
\text { lesions } \\
(n=44)\end{array}$ & $\begin{array}{l}\text { External GW } \\
(n=47)\end{array}$ & $\begin{array}{l}\text { No HPV Disease } \\
(n=43)\end{array}$ & Overall & $P$-value* \\
\hline \multicolumn{8}{|l|}{ HPV Impact Profile Total Score } \\
\hline Mean & 44.81 & $40.69^{1}$ & 50.52 & $55.84^{1}$ & 48.06 & 47.99 & \multirow[t]{3}{*}{0.0040} \\
\hline SD & 20.09 & 18.29 & 19.47 & 18.71 & 20.56 & 19.94 & \\
\hline $95 \% \mathrm{Cl}$ & $(38.8 ; 50.8)$ & $(35.2 ; 46.2)$ & $(44.6 ; 56.4)$ & $(50.3 ; 61.4)$ & $(41.7 ; 54.5)$ & $(45.4 ; 50.6)$ & \\
\hline \multicolumn{8}{|l|}{ Worries and Concerns } \\
\hline Mean & 43.77 & $41.71^{1}$ & 54.06 & $58.72^{1}$ & 49.11 & 49.47 & \multirow[t]{3}{*}{0.0178} \\
\hline SD & 27.37 & 27.08 & 25.75 & 25.24 & 30.74 & 27.75 & \\
\hline $95 \% \mathrm{Cl}$ & $(35.6 ; 51.9)$ & $(33.6 ; 49.8)$ & $(46.2 ; 61.9)$ & $(51.2 ; 66.2)$ & $(39.5 ; 58.7)$ & $(45.8 ; 53.1)$ & \\
\hline \multicolumn{8}{|l|}{ Emotional Impact } \\
\hline Mean & 45.17 & $35.16^{1}$ & 48.49 & $57.66^{1}$ & 45.62 & 46.52 & \multirow[t]{3}{*}{0.0004} \\
\hline SD & 24.14 & 24.22 & 24.40 & 21.24 & 24.71 & 24.64 & \\
\hline $95 \% \mathrm{Cl}$ & $(38.0 ; 52.3)$ & $(27.9 ; 42.4)$ & $(41.1 ; 55.9)$ & $(51.4 ; 63.9)$ & $(37.9 ; 53.3)$ & $(43.3 ; 49.8)$ & \\
\hline \multicolumn{8}{|l|}{ Sexual Impact } \\
\hline Mean & 47.33 & 43.30 & 51.59 & 53.78 & 51.58 & 49.47 & \multirow[t]{3}{*}{0.1738} \\
\hline SD & 23.47 & 20.54 & 22.43 & 22.77 & 19.77 & 22.03 & \\
\hline $95 \% \mathrm{Cl}$ & $(40.3 ; 54.4)$ & $(37.0 ; 49.5)$ & $(44.8 ; 58.4)$ & $(46.9 ; 60.6)$ & $(45.1 ; 58.1)$ & $(46.5 ; 52.4)$ & \\
\hline \multicolumn{8}{|l|}{ Self-image } \\
\hline Mean & 41.30 & 41.44 & 41.88 & 49.60 & 44.31 & 43.72 & \multirow[t]{3}{*}{0.2115} \\
\hline SD & 19.91 & 18.03 & 20.38 & 18.44 & 21.09 & 19.66 & \\
\hline $95 \% \mathrm{Cl}$ & $(35.4 ; 47.2)$ & $(36.0 ; 46.9)$ & $(35.7 ; 48.1)$ & $(44.1 ; 55.1)$ & $(37.7 ; 50.9)$ & $(41.1 ; 46.3)$ & \\
\hline \multicolumn{8}{|l|}{ Partner Issues and Transmission } \\
\hline Mean & $49.71^{1}$ & $47.67^{2}$ & 57.46 & $66.22^{1,2}$ & 54.91 & 55.22 & \multirow[t]{3}{*}{0.0069} \\
\hline SD & 26.37 & 24.77 & 26.33 & 23.12 & 27.37 & 26.19 & \\
\hline $95 \% \mathrm{Cl}$ & $(41.9 ; 57.5)$ & $(40.1 ; 55.3)$ & $(49.3 ; 65.7)$ & $(59.3 ; 73.2)$ & $(45.6 ; 64.2)$ & $(51.7 ; 58.8)$ & \\
\hline \multicolumn{8}{|l|}{ Interactions with Doctors } \\
\hline Mean & 41.52 & $31.04^{1}$ & $46.36^{2}$ & $52.07^{1,2}$ & 41.30 & 42.46 & \multirow[t]{3}{*}{0.0004} \\
\hline SD & 22.07 & 18.61 & 24.26 & 25.14 & 22.05 & 23.41 & \\
\hline $95 \% \mathrm{Cl}$ & $(35.0 ; 48.1)$ & $(25.4 ; 36.6)$ & $(39.0 ; 53.7)$ & $(44.5 ; 59.6)$ & $(34.3 ; 48.3)$ & $(39.4 ; 45.6)$ & \\
\hline \multicolumn{8}{|l|}{ Control/Life Impact } \\
\hline Mean & 48.26 & 47.11 & 51.74 & 52.16 & 54.88 & 50.77 & \multirow[t]{3}{*}{0.2894} \\
\hline SD & 19.02 & 16.58 & 17.56 & 22.09 & 16.88 & 18.64 & \\
\hline $95 \% \mathrm{Cl}$ & $(42.6 ; 53.9)$ & $(42.1 ; 52.1)$ & $(46.4 ; 57.1)$ & $(45.7 ; 58.6)$ & $(49.6 ; 60.1)$ & $(48.3 ; 53.2)$ & \\
\hline \multicolumn{8}{|c|}{ HPV Impact Profile Total Score categorized } \\
\hline No or little impact & $20(43.5 \%)$ & $22(48.9 \%)$ & $12(27.3 \%)$ & $10(21.3 \%)$ & 15 (34.9\%) & 79 (35.1\%) & \\
\hline Moderate impact & $20(43.5 \%)$ & $21(46.7 \%)$ & $24(54.5 \%)$ & $23(48.9 \%)$ & $21(48.8 \%)$ & $109(48.4 \%)$ & \\
\hline Heavy psychological impact & $6(13.0 \%)$ & $2(4.4 \%)$ & $8(18.2 \%)$ & $14(29.8 \%)$ & 7 (16.3\%) & 37 (16.4\%) & \\
\hline Valid n & 46 & 45 & 44 & 47 & 43 & 225 & \\
\hline
\end{tabular}

*Analysis of Variance (ANOVA) test

Equal numbers show statistical differences between these means at level 0.05

Cl confidence interval, GW genital warts, HPV human papillomavirus, Pap Papanicolaou test, SD standard deviation 


\section{Abbreviations}

CECA: Cuestionario Especifico para Condiloma Acuminado; Cl: confidence interval; CIN: cervical intraepithelial neosplasia; DERM: Dermatologist; EQ5D: EuroQol-5 Dimension; ER: emergency room; FM: family medicine; GP: general practitioner; GW: genital warts; HIP: HPV impact profile; HPV: human papillomavirus; HRQL: health related quality of life; IDS: infectious disease specialist; IMS: Intercontinental Marketing Services; OB/ GYN: obstetrician/gynecologist; Pap: Papanicolaou; PCP: primary care physician; PHP: Philippine peso; SD: standard deviation; STI: sexually transmitted infection; TPP: third party payer; URO: urologist; VAS: Visual Analogue Scale

\section{Acknowledgements}

Not applicable.

\section{Authors' contributions}

SK, AK, and KY conceptualized and designed the study, contributed to the interpretation of the data, and substantially contributed to critical revisions of the intellectual content. NL and MR contributed to the acquisition and interpretation of the data and substantially contributed to critical revisions of the intellectual content. LB, ARG, and SG contributed to the interpretation of the data and substantially contributed to critical revisions of the intellectual content. All authors read and approved the final manuscript.

\section{Funding}

Merck \& Co funded the study and also participated in the interpretation of the data and the writing of the manuscript.

\section{Availability of data and materials}

The data analyzed during the current study are available from the corresponding author on reasonable request.

\section{Ethics approval and consent to participate}

Ethical approval was obtained from an accredited external Institutional Review Board (IRB) for those sites not covered by their own internal IRB. The ethical conduct of this study was performed in accordance with the Declaration of Helsinki and the principles of Good Clinical Practices.

\section{Consent for publication}

Not applicable.

\section{Competing interests}

LB declares they have no conflicts to disclose.

AK and SKT are employees of Merck \& Co.

SMG received grants to her institution from: the Commonwealth Department of Health for human papillomavirus (HPV) genoprevalence surveillance post-vaccination; Merck \& Co. and GSK to perform phase 3 clinical vaccine trials; Merck \& Co. to evaluate HPV in a post-vaccination program; Commonwealth Serum Laboratories for an HPV in cervical cancer study; and the Veterinary Centers of America for a study on the effectiveness of a public health HPV vaccine and a study on the associations of early onset cancers. SMG also received speaking fees from Merck Sharp and Dohme Corp., and Sanofi Pasteur/Merck Sharp and Dohme for work performed in her personal time. Merck \& Co. paid for travel and accommodations to present at HPV Advisory board meetings.

KY was a paid contractor for Merck \& Co. at the time of the study and was an employee of Cubist Pharmaceuticals from December 2014 to July 2015, which was acquired by Merck \& Co. in January 2015.

ARG is a member of Merck \& Co. advisory boards. Her institution has received grants and contracts to support HPV-related research. NL and MR are employees of IMS Health, Barcelona, Spain, which is a paid consultant to Merck \& Co.

\section{Author details}

'Makati Medical Center, Makati, Philippines. ${ }^{2}$ Merck \& Co. Inc., Kenilworth, NJ, USA. ${ }^{3}$ Cubist Pharmaceuticals, Lexington, MA, USA. ${ }^{4}$ IMS Health, Barcelona, Spain. ${ }^{5}$ Center for Infection Research in Cancer (CIRC) at Moffitt Cancer Center, Tampa, FL, USA. ${ }^{6}$ Royal Women's Hospital, Melbourne, Australia.
Received: 21 February 2019 Accepted: 22 August 2019

Published online: 07 October 2019

\section{References}

1. Arima Y, Winer RL, Feng Q, Hughes JP, Lee SK, Stern ME, et al. Development of genital warts after incident detection of human papillomavirus infection in young men. J Infect Dis. 2010;202(8):1181-4.

2. Taylor LSD, Hariri S, Sternberg M, Dunne EF, Markowitz LE. Human papillomavirus vaccine coverage in the United States, National Health and nutrition examination survey, 2007-2008. Prev Med. 2011;52(5):398-400.

3. Haupt RM, Sings HL. The efficacy and safety of the quadrivalent human papillomavirus 6/11/16/18 vaccine gardasil. J Adolesc Health. 2011;49(5):467-75.

4. Garland SM, Steben M, Sings HL, James M, Lu S, Railkar R, et al. Natural history of genital warts: analysis of the placebo arm of 2 randomized phase III trials of a quadrivalent human papillomavirus (types $6,11,16$, and 18) vaccine. J Infect Dis. 2009;199(6):805-14.

5. Kim MA, Oh JK, Kim BW, Chay D, Park DC, Kim SM, et al. Prevalence and seroprevalence of low-risk human papillomavirus in Korean women. J Korean Med Sci. 2012;27(8):922-8.

6. Raymakers AJ, Sadatsafavi M, Marra F, Marra CA. Economic and humanistic burden of external genital warts. Pharmacoeconomics. 2012;30(1):1-16.

7. Castellsagué X, Cohet C, Puig-Tintoré L, Acebes LO, Salinas J, San Martin M, et al. Epidemiology and cost of treatment of genital warts in Spain. Eur J Pub Health. 2009;19(1):106-10.

8. Graziottin A, Serafini A. HPV infection in women: psychosexual impact of genital warts and intraepithelial lesions. J Sex Med. 2009;6(3):633-45.

9. Clifford GM, Gallus S, Herrero R, Muñoz N, Snijders PJF, Vaccarella S, et al. Worldwide distribution of human papillomavirus types in cytologically normal women in the International Agency for Research on Cancer HPV prevalence surveys: a pooled analysis. Lancet. 2005;366:991-8.

10. Dinh TH, Sternberg M, Dunne EF, Markowitz LE. Genital warts among 18- to 59-year-olds in the United States, National Health and nutrition examination survey, 1999-2004. Sex Transm Dis. 2008;35(4):357-60.

11. Hartwig S, St Guily JL, Dominiak-Felden G, Alemany L, de Sanjosé S. Estimation of the overall burden of cancers, precancerous lesions, and genital warts attributable to 9-valent HPV vaccine types in women and men in Europe. Infect Agents Cancer. 2017;12(1):19.

12. Pirotta M, Stein AN, Conway EL, Harrison C, Britt H, Garland S. Genital warts incidence and healthcare resource utilisation in Australia. Sex Transm Infect. 2010;86(3):181-6.

13. Harrison C, Britt H, Garland S, Conway L, Stein A, Pirotta M, et al. Decreased management of genital warts in young women in Australian general practice post introduction of National HPV vaccination program: Results from a nationally representative cross-sectional general practice study. PLoS One. 2014;9(9):e10596.

14. Pirotta MV, Stein AN, Fairley CK, Morrow A, Conway EL, Chuah J, et al. Patterns of treatment of external genital warts in Australian sexual health clinics. Sex Transm Dis. 2009;36(6):375-9.

15. Lin C, Lau J, Ho KM, Lau MC, Tsui HY, Lo KK. Incidence of genital warts among the Hong Kong general adult population. BMC Infect Dis. 2010; $10: 272$.

16. Hillemanns P, Breugelmans JG, Gieseking F, Bénard S, Lamure E, Littlewood $\mathrm{KJ}$, et al. Estimation of the incidence of genital warts and the cost of illness in Germany: a cross-sectional study. BMC Infect Dis. 2008;8(1):76.

17. Tsai TF, Kothari-Talwar S, Yee K, Kulkarni A, Lara N, Roset M, et al. Estimating the burden of genital warts in Taiwan. Sex Health. 2017;14(6):485-91.

18. Tan LS, Chio MT, Sen P, Lim YK, Ng J, llancheran A, et al. Assessment of psychosocial impact of genital warts among patients in Singapore. Sex Health. 2014;11(4):313-8.

19. Qi SZ, Wang SM, Shi JF, Wang QQ, Chen XS, Sun LJ, et al. Human papillomavirus-related psychosocial impact of patients with genital warts in China: a hospital-based cross-sectional study. BMC Public Health. 2014;14:739.

20. Wang SM, Shi JF, Kang DJ, Song P, Qiao YL. Impact of human papillomavirus-related lesions on quality of life: a multicenter hospital-based study of women in mainland China. Int J Gynecol Cancer. 2011;21(1):182-8.

21. Ngelangel CA, Wang EH. Cancer and the Philippine cancer control program. Jpn J Clin Oncol. 2002;32(suppl 1):S52-61.

22. Miyashita M, Agdamag DM, Sasagawa T, Matsushita K, Salud LM, Salud CO, et al. High-risk HPV types in lesions of the uterine cervix of female commercial sex workers in the Philippines. J Med Virol. 2009;81(3):545-51. 
23. Mast TC, Zhu X, Demuro-Mercon C, Cummings HW, Sings HL, Ferris DG. Development and psychometric properties of the HPV impact profile (HIP) to assess the psychosocial burden of HPV. Curr Med Res Opin. 2009;25(11): 2609-19.

24. Badia X, Colombo JA, Lara N, Llorens MA, Olmos L, Sainz de los Terreros M, et al. Combination of qualitative and quantitative methods for developing a new health related quality of life measure for patients with anogenital warts Health Qual Life Outcomes 2005;3:24.

25. Vilata JJ, Varela JA, Olmos L, Colombo JA, Llorens MA, Sainz De Los Terreros $M$, et al. ECCAVIM study group. Validation and clinical use of the CECA, a disease-specific quality of life questionnaire for patients with anogenital condylomata acuminata. Acta Derm Venereol. 2008;88(3):257-62.

26. EuroQol Group. EuroQol--a new facility for the measurement of healthrelated quality of life. Health Policy. 1990;16:199-208.

27. Tsuchiya A, Ikeda S, Ikegami N, Nishimura S, Sakai I, Fukuda T, et al. Estimating an EQ-5D population value set: the case of Japan. Health Econ. 2002;11(4):341-53.

28. Sakthong P, Charoenvisuthiwongs R, Shabunthom R. A comparison of EQ5D index scores using the UK, US, and Japan preference weights in a Thai sample with type 2 diabetes. Health Qual Life Outcomes. 2008;6:71.

29. Vora R, Anjaneyan G, Doctor C, Gupta R. Clinico-epidemiological study of sexually transmitted infections in males at a rural-based tertiary care center. Indian J Sex Transmis Dis. 2011;32:86-9.

30. Hoy T, Singhal PK, Willey VJ, Insinga RP. Assessing incidence and economic burden of genital warts with data from a US commercially insured population. Curr Med Res Opin. 2009;25(10):2343-51.

31. Kjaer SK, Tran TN, Sparen P, Tryggvadottir L, Munk C, Dasbach E, et al. The burden of genital warts: a study of nearly 70,000 women from the general female population in the 4 Nordic countries. J Infect Dis. 2007;196(10):1447-54.

32. García PJ, Carcamo CP, Valderrama M, La Rosa S, James C, Gutiérrez R, et al. Burden of genital warts in Peru: an observational study. Int J STD AIDS. 2019;30(3):264-74.

33. Klavs I, Grgic-Vitek M. The burden of genital warts in Slovenia: results from a national probability sample survey. Euro Surveillance. 2008;13(45):19032.

34. Baseman JG, Koutsky LA. The epidemiology of human papillomavirus infections. J Clin Virol. 2005;32(Suppl 1):16-24.

35. Lee CB, Choe HS, Hwang SJ, Lee SJ, Cho YH. Epidemiological characteristics of genital herpes and condyloma acuminata in patients presenting to urologic and gynecologic clinics in Korea. J Infect Chemother. 2011;17(3):351-7.

36. Lee TS, Kothari-Talwar S, Singhal PK, Yee K, Kulkarni A, Lara N, et al. A crosssectional study estimating the burden of illness related to genital warts in South Korea. BMJ Open. 2017;7(6):e014217.

37. Domenech-Viñolas M, León-Maldonado L, Ramírez-Palacios P, Flores YN, Granados-García V, Brown B, et al. Incidence, psychosocial burden, and economic impact of genital warts in Mexico. Salud Publica Mex. 2018; 60(6):624-32.

38. Trading Economicse, Philippine GDP per Capita. https://tradingeconomics. com/philippines/gdp-per-capita. Accessed 24 Jan 2018.

39. Philippine Statistics Authority, 2012 and 2015 Family Income and Expenditure Survey. https://psa.gov.ph/sites/default/files/attachments/hsd/ article/tab2.pdf. Accessed 25 Jan 2018

40. Mrus JM, Williams PL, Tsevat J, Cohn SE, Wu AW. Gender differences in health-related quality of life in patients with HIV/AIDS. Qual Life Res. 2005; 14(2):479-91.

41. French $\subset T$, Fletcher $K E$, Irwin RS. Gender differences in health-related quality of life in patients complaining of chronic cough. Chest. 2004;125(2):182-8.

42. Emery CF, Frid DJ, Engebretson TO, Alonzo AA, Fish A, Ferketich AK, et al. Gender differences in quality of life among cardiac patients. Psychosom Med. 2004;66(2):190-7.

43. Raj R, Sreenivs V, Mehta M, Gupta S. Health-related quality of life in Indian patients with three viral sexually transmitted infections: herpes simplex virus-2, genital human papilloma virus and HIV. Sex Transm Infect. 2011:87:216-20

44. Lee SJ, Yun HJ, Lee KH, Kim CJ, Park JS. What questions do people ask on a human papilloma website? A comparative analysis of public and private questions. Int J Med Sci. 2012;9(2):142-7.

45. Sénécal M, Brisson M, Maunsell E, Ferenczy A, Franco EL, Ratnam S, et al. Loss of quality of life associated with genital warts: baseline analyses from a prospective study. Sex Transm Infect. 2011;87(3):209-15.

46. Wang KL, Shi JF, Kang DJ, Song P, Qiao YL. Chinese HPV study group Impact of human papillomavirus-related lesions on quality of life: a multicenter hospital-based study of women in Mainland China. Int J Gynecol Cancer. 2011;21:182-8.

47. Wang KL, Jeng CJ, Yang YC, Chen CA, Cheng WF, Chen TC, et al. The psychological impact of illness among women experiencing human papillomavirus-related illness or screening interventions. J Psychosom Obstet Gynaecol. 2010;31(1):16-23.

\section{Publisher's Note}

Springer Nature remains neutral with regard to jurisdictional claims in published maps and institutional affiliations.
Ready to submit your research? Choose BMC and benefit from:

- fast, convenient online submission

- thorough peer review by experienced researchers in your field

- rapid publication on acceptance

- support for research data, including large and complex data types

- gold Open Access which fosters wider collaboration and increased citations

- maximum visibility for your research: over $100 \mathrm{M}$ website views per year

At $\mathrm{BMC}$, research is always in progress.

Learn more biomedcentral.com/submissions 